\title{
BCKDK regulates the TCA cycle through PDC to ensure embryonic development in the absence of PDK family
}

Lia Heinemann-Yerushalmi, ${ }^{* 1}$ Lital Bentovim, ${ }^{* 1}$ Neta Felsenthal, ${ }^{1}$ Ron Carmel Vinestock, ${ }^{1}$ Nofar Michaeli, ${ }^{1}$ Sharon Krief, ${ }^{1}$ Alon Silberman, ${ }^{5}$ Marina Cohen, ${ }^{2}$ Shifra Ben-Dor, ${ }^{3}$ Ori Brenner, ${ }^{2}$ Rebecca Haffner-Krausz, ${ }^{2}$ Maxim Itkin, ${ }^{4}$ Sergey Malitsky, ${ }^{4}$ Ayelet Erez ${ }^{5}$ and Elazar Zelzer ${ }^{1}$

${ }^{1}$ Department of Molecular Genetics, Weizmann Institute of Science, 234 Herzl St, Rehovot 7610001, Israel

${ }^{2}$ Department of Veterinary Resources, Weizmann Institute of Science, 234 Herzl St, Rehovot 7610001, Israel

${ }^{3}$ Bioinformatics and Biological Computing Unit, Biological Services, Weizmann Institute of Science, Rehovot, 76100, Israel

${ }^{4}$ Department of Life Sciences Core Facilities, Weizmann Institute of Science, Rehovot, Israel

${ }^{5}$ Department of Biological regulation, Weizmann Institute of Science, Rehovot, Israel *These authors equally contributed to this paper.

\section{Correspondence:}

Elazar Zelzereli.zelzer@weizmann.ac.il 


\begin{abstract}
Pyruvate dehydrogenase kinases (PDK1-4) inhibit the TCA cycle by phosphorylating pyruvate dehydrogenase complex (PDC). Here, we show that the PDK family is dispensable for the survival of murine embryonic development and that BCKDK serves as a compensatory mechanism by inactivating PDC.

First, we knocked out all four $P d k$ genes one by one. Surprisingly, $P d k$ total $\mathrm{KO}$ embryos developed and were born in expected ratios, but died by postnatal day 4 due to hypoglycemia or ketoacidosis.

Finding that PDC was phosphorylated in these embryos suggested that another kinase compensates for the PDK family. Bioinformatic analysis implicated brunch chain ketoacid dehydrogenase kinase (Bckdk), a key regulator of branched chain amino acids (BCAA) catabolism. Indeed, knockout of $B c k d k$ and the $P d k$ family led to loss of PDC phosphorylation, increment in PDC activity, elevation of Pyruvate flux into the TCA and early embryonic lethality. These findings reveal a new regulatory crosstalk hardwiring BCAA and glucose catabolic pathways, which feed the TCA cycle.
\end{abstract}




\section{Introduction}

The pyruvate dehydrogenase complex (PDC) plays a major role as the gatekeeper that links glycolysis to the TCA cycle, maintaining metabolic balance and energy production through the rate-limiting and physiologically irreversible oxidative decarboxylation of pyruvate ${ }^{1}$. Due to its importance, PDC regulation has been extensively studied since its discovery in the late 1960's ${ }^{2}$. The regulatory mechanism was shown to involve reversible phosphorylation by intrinsic regulatory enzymes, a family of pyruvate dehydrogenase kinases (PDK) $1-4^{3}$. These isoenzymes phosphorylate and inactivate PDC on three serine residues of its catalytic E1 $\alpha$ subunit (PDH1a), namely S293 (site 1), S300 (site 2), and S232 (site 3). Studies of site specificity showed that all four PDKs phosphorylate site 1 and site 2, whereas site 3 is phosphorylated only by PDK1. Yet, phosphorylation of any of the three serine residues leads to complete inactivation of PDC activity ${ }^{4}$.

PDK activity blocks the flux of pyruvate into the TCA cycle, which results in a metabolic shift to glycolysis for energy production. PDC activity is regulated by Pdk family in a short and long-term manner ${ }^{5}$. In the short term, several allosteric regulators can activate PDK according to the levels of end products, namely increased acetyl-CoA/coenzyme A ratio and reduced and oxidized nicotinamide adenine dinucleotide $\left(\mathrm{NADH} / \mathrm{NAD}^{+}\right)$ratio $^{6}$. In the long term, the amount of PDK protein is determined by different physiological conditions and in a tissue-specific manner ${ }^{7-10}$. This involves transcriptional and translational regulation via hormonal regulators, such as estrogen receptor and glucocorticoid receptor ${ }^{11}$, and transcription factors such as oxygen sensor hypoxia inducible factor 1 subunit alpha $(\text { HIF } 1 \alpha)^{12-18}$. HIF $1 \alpha$ regulation of $P d k$ was shown to occur in cancer cells, which prefer to maintain anaerobic metabolism even in the presence of oxygen, a phenomenon known as the Warburg effect or aerobic glycolysis ${ }^{19}$. Here, the increase in glycolysis supplies 
intermediates for branching pathways that synthesize the macromolecules necessary for cell proliferation ${ }^{20}$. Based on these studies, $P d k$ family and specifically PDK1 became a specific target for anti-cancer drug development ${ }^{5,21}$. In addition to its role in cancer, the HIF1-PDK axis is also vital for mammalian embryonic development ${ }^{22-24}$. For example, during endochondral bone formation, HIF1 $\alpha$ is required for various processes in the hypoxic growth plate ${ }^{25-29}$, one of which is to directly regulate the expression of $P d k 1^{30}$. Extensive studies on the involvement of the $P d k$ family in metabolic regulation have provided some indications for functional redundancy among its members. Cell culture experiments showed redundancy between PDK1 and PDK2 ${ }^{31}$, whereas loss-of-function studies in mice lacking Pdk2, Pdk4 or even both revealed no major effect ${ }^{31-33}$. Nevertheless, the question of the necessity of the four PDK isoenzymes and the functional redundancy among them has yet to be addressed by a direct genetic approach.

In this study, we investigated the requirement of Pdk's for embryonic development using the hypoxic growth plate as a model. Strikingly, mouse strains of double, triple and eventually quadruple KO, i.e. mice lacking all four $P d k$ genes, displayed normal skeletal development and were born in the expected Mendelian ratios. These results suggest that the entire $P d k$ family is dispensable for embryogenesis. Moreover, we show for the first time that in the absence of all PDK isoenzymes, PDC is still phosphorylated, implying the existence of a backup mechanism. Bioinformatic analysis implicated BCKDK, an enzyme that regulates the catabolism of branch chain amino acids, in this mechanism. The loss of PDC phosphorylation and consequent increases in PDC activity and pyruvate flux into the TCA cycles, which we observed in mice and cell lines lacking all $P d k$ 's and $B c k d k$, strongly support this possibility. Overall, we identify BCKDK regulation of PDC as a novel mechanism that can backup PDK family function to maintain embryonic development. 


\section{RESULTS}

\section{PDK1, PDK2 and PDK4 are dispensable for development and growth}

To study in vivo the role of PDK family, we first focused on PDK1. Since the involvement of this isoenzyme in metabolic homeostasis was mostly studied in the context of cells under hypoxic conditions ${ }^{14,15,34,35}$, we chose the hypoxic growth plate during mouse bone development as a model ${ }^{25,26,29,30,36}$. Using the knockout-first allele method, we generated three mouse strains: floxed-Pdk1, Pdkl-lacZ knockout (KO) and Pdk1 KO (Fig. S1A). Examination of the Pdkl-lacZ KO reporter line verified strong $P d k 1$ expression in developing bones of E14.5 embryos, including long bones, vertebrae and facial bones (Fig. S1B-C). Surprisingly, both homozygous Pdkl-lacZ KO and Pdk1 KO mice had no apparent bone phenotype during embryonic development, as confirmed by skeletal preparation (Fig. 1A,B) and histological sections (Fig. 1C,D). These mice produced viable and fertile colonies matching expected Mendelian ratios (Fig. 1G).

To understand the lack of phenotype, we examined the levels of different metabolites related to the TCA and glycolysis pathways in growth plate chondrocytes from $P d k 1 \mathrm{KO}$ and control mice. Chondrocytes from tibiofemoral growth plates were extracted and grown in cell culture, and the TCA and glycolysis intermediates were examined. Results showed similar levels of metabolites (glucose, glutamine and glutamate) in control and Pdkl $\mathrm{KO}$ chondrocytes, indicating that metabolic homeostasis in those cells is maintained. Moreover, we detected similar levels of lactate, suggesting that a metabolic shift to enhanced glycolysis occurred in the absence of $P d k 1$ (Fig. 1F).

A plausible explanation for the lack of phenotype in the absence of $P d k 1$ is that other PDK isoenzymes compensated for its activity. Null mutants for $P d k 2$ and $P d k 4$ were reported to 
develop normally and have viable and fertile colonies ${ }^{31,32}$. Therefore, to examine the functional redundancy hypothesis, we generated double $\mathrm{KO}(\mathrm{dKO})$ and triple $\mathrm{KO}(\mathrm{tKO})$ mouse models lacking $P d k 1$ and $P d k 2$, or $P d k 1$ and $P d k 4$, or all three of these genes. The loss of these genes was verified by quantitative RT-PCR (qRT PCR) (Fig. S2A). Surprisingly, none of the combinations exhibited any bone phenotype (Fig. 1H-M). Moreover, Pdk tKO mice produced viable and fertile colonies matching expected Mendelian ratios (Fig. 1N). These outcomes indicated that PDK1, PDK2 and PDK4 are dispensable for bone and embryonic development. 

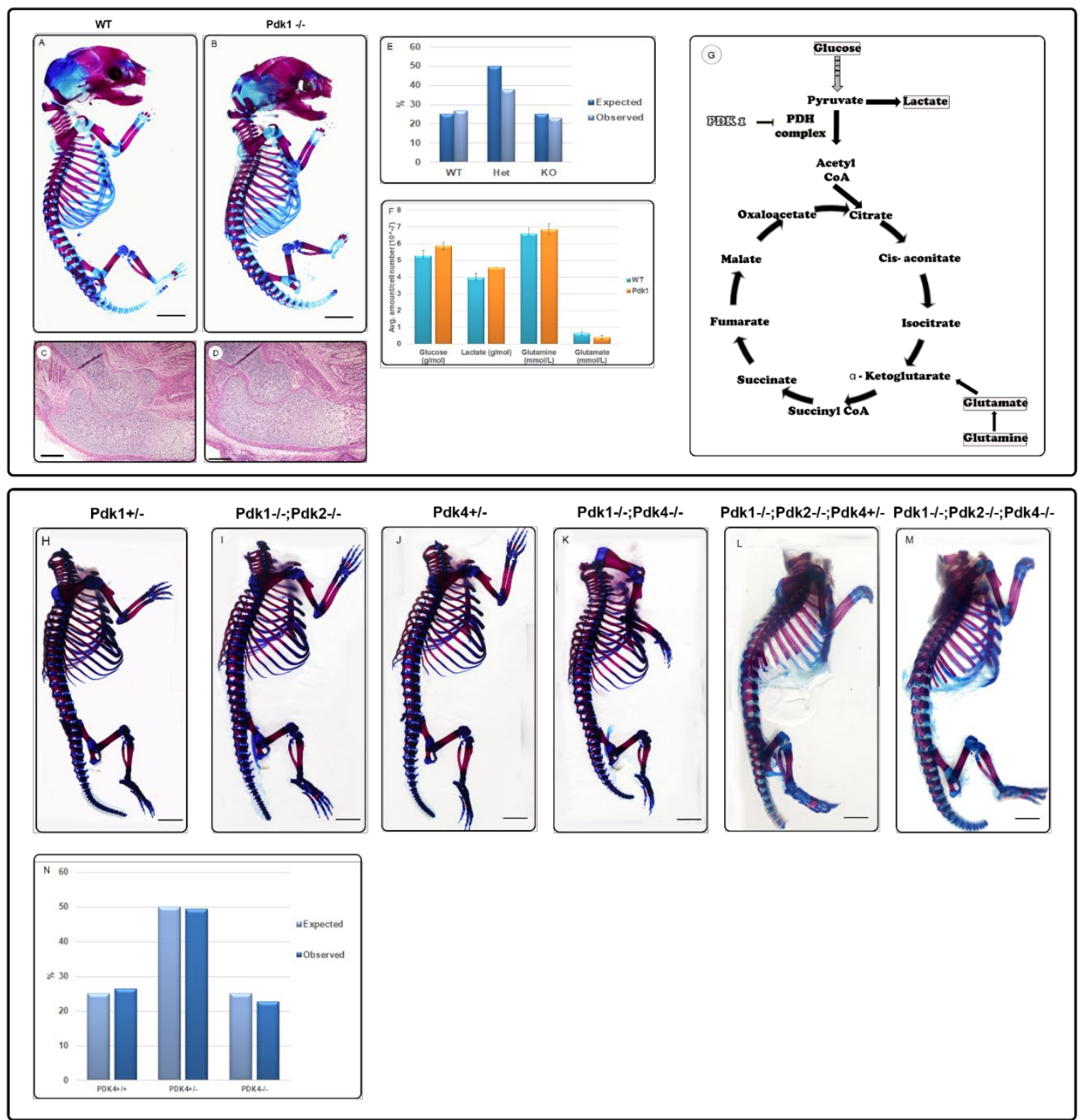

Figure 1. Deletion of single, double or triple $P d k$ genes in vivo does not affect embryonic development. (A,B) Skeletal preparations of control (A) and Pdk1 KO (B) E17.5 embryos showing no skeletal phenotype (scale, $50 \mu \mathrm{m})$. (C,D) H\&E staining of control and Pdk1 KO E15.5 embryonic sections of olecranon and distal humerus growth plates (scale, $50 \mu \mathrm{m}$ ). (E) Graph showing expected Mendelian ratios of Pdk1 KO progeny. 
(F) Graph showing similar absolute levels of glycolysis-TCA pathway-related metabolites in chondrocytes from E17.5 Pdk1 KO and WT embryos (Nova analyzer, n=3). Schematic illustration of the TCA cycle highlighting the relevant metabolites entry point to the cycle . (H-M) Skeletal preparations of control $\left(P d k 1^{+/}, \mathrm{H}\right), P d k 1-P d k 2 \mathrm{dKO}(\mathrm{I})$, control $\left(P d k 4^{+/-}, \mathrm{J}\right)$ and Pdkl-Pdk4 Dko $(\mathrm{K})$, control $\left(P d k 1^{-/-} P d k 2^{-/-} P d k 4^{+/-}, 1\right)$ and $P d k 1-$ Pdk2-Pdk4 tKO (M) newborn pups. (H and I, J and K, and L and M are littermates; scale, $50 \mu \mathrm{m})$. (N) Graph showing expected Mendelian ratios in colonies of $P d k$ tKO mice.

\section{Loss of the Pdk family results in postnatal lethality due to hypoglycemia and ketoacidosis}

The lack of phenotype in the $P d k$ tKO mice led us to study the only remaining candidate from the $P d k$ family, namely $P d k 3$. Recently, $P d k 3$ was also shown to be a HIF $1 \alpha$ target under hypoxic conditions ${ }^{18}$. Thus, we hypothesized that $P d k 3$ might be sufficient to maintain embryonic development. To test this hypothesis, we established a genetic model in which the expression of all four $P d k$ genes is deleted. Considering the possible embryonic lethality, we sought to delete $P d k 3$ on the background of an intact $P d k 1$ using $P d k 1^{f l o x f l o x}$ allele combined with $P d k 2$ and $P d k 4$ KO. By crossing these mice with a specific Cre line, we could remove $P d k l$ in a tissue-specific manner and, thereby, prevent early lethality. For that purpose, we utilized the CRISPR/Cas9 method to target $P d k 3$ gene on the genetic background of Prxl-Cre;Pdk1 flox/null $P d k 2^{-/} P d k 4^{-/-}$mice and generated two independent lines. The first was the conditional quadruple knockout (cKO) PrxlCre; $P d k 1^{\text {floxfllox }} P d k 2^{-/-} P d k 3^{-/} P d k 4^{-/}$mice, which lack all four Pdk genes in Prxl-expressing limb mesenchyme lineages only. The second line was back-crossed to generate $P d k 1^{-/-}$ $P d k 2^{-/-} P d k 3^{-/-} P d k 4^{-/-}$quadruple $\mathrm{KO}$ mice (total KO) (Fig. 2A). 
Using qRT-PCR and western blot analysis on $P d k$ total KO mice, we verified deletion of all four Pdk genes at both mRNA and protein levels (Fig. 2B,C). Moreover, western blot analysis of chondrocytes derived from Pdk cKO growth plates showed deletion of PDK1 protein compared to cells from WT and control littermates, where Prx1-Cre was not present (Fig. 2D). The results indicated effective deletion of all four $P d k$ genes in both total $\mathrm{KO}$ and cKO mice. Surprisingly, skeletal preparation and histological sections from $P d k$ cKO mice revealed no bone phenotype (Fig. 2E-F'). Monitoring the colonies, we found that $P d k$ cKO mice produced viable and fertile colonies matching expected Mendelian ratios (Fig. 2G). Similarly, examination of bone development in skeletal preparation and histological sections of $P d k$ total $\mathrm{KO}$ embryos revealed no major skeletal phenotype (Fig. 2H-I'). However, although embryonic Mendelian ratios were observed as expected (Fig. $2 \mathrm{~J})$, this mouse strain did not produce viable offspring, as shown by the non-Mendelian ratios of mature mice (Fig. $2 \mathrm{~K}$ ).

Examination of newborn $P d k$ total KO pups revealed that they died between P0 and P4. To determine the cause of death, we analyzed histological sections from adrenal gland, brain, brown fat tissue, heart, kidney, liver, lung, small intestines and spinal cord of E18.5 embryos. The examination revealed no major anatomical abnormalities (Fig. S3).

Previous study showed that $P d k$ loss results in high utilization of glucose, followed by elevation of ketone bodies ${ }^{33}$. We therefore examined blood levels of glucose and ketone bodies as a possible cause of lethality. As observed in Fig. 2L-M, whereas at E18.5 no differences where observed between WT and Pdk total KO embryos, P1 Pdk total KO pups displayed significantly higher levels of 3- $\beta$-hydroxybutyrate (3HB), a common ketone body, and lower glucose levels as compared to WT pups (Fig. 2M). 
Deeper metabolic profiling of serum composition in these pups showed significantly low levels of pyruvate and lactate together with a higher utilization of acetyl CoA, reflected by higher levels of the ketone bodies acetoacetate and 3HB, reduced production of more distal TCA metabolites, and high levels of branched chain amino acids (BCAA) (Fig. 2N). These results are consistent with the expected effect of $P d k$ loss ${ }^{33,37}$, and suggest that the cause of the early postnatal death was ketoacidosis and hypoglycemia. Altogether, these results suggest that $P d k$ family is dispensable for embryonic development but is necessary postnatally to maintain energy metabolic balance. 


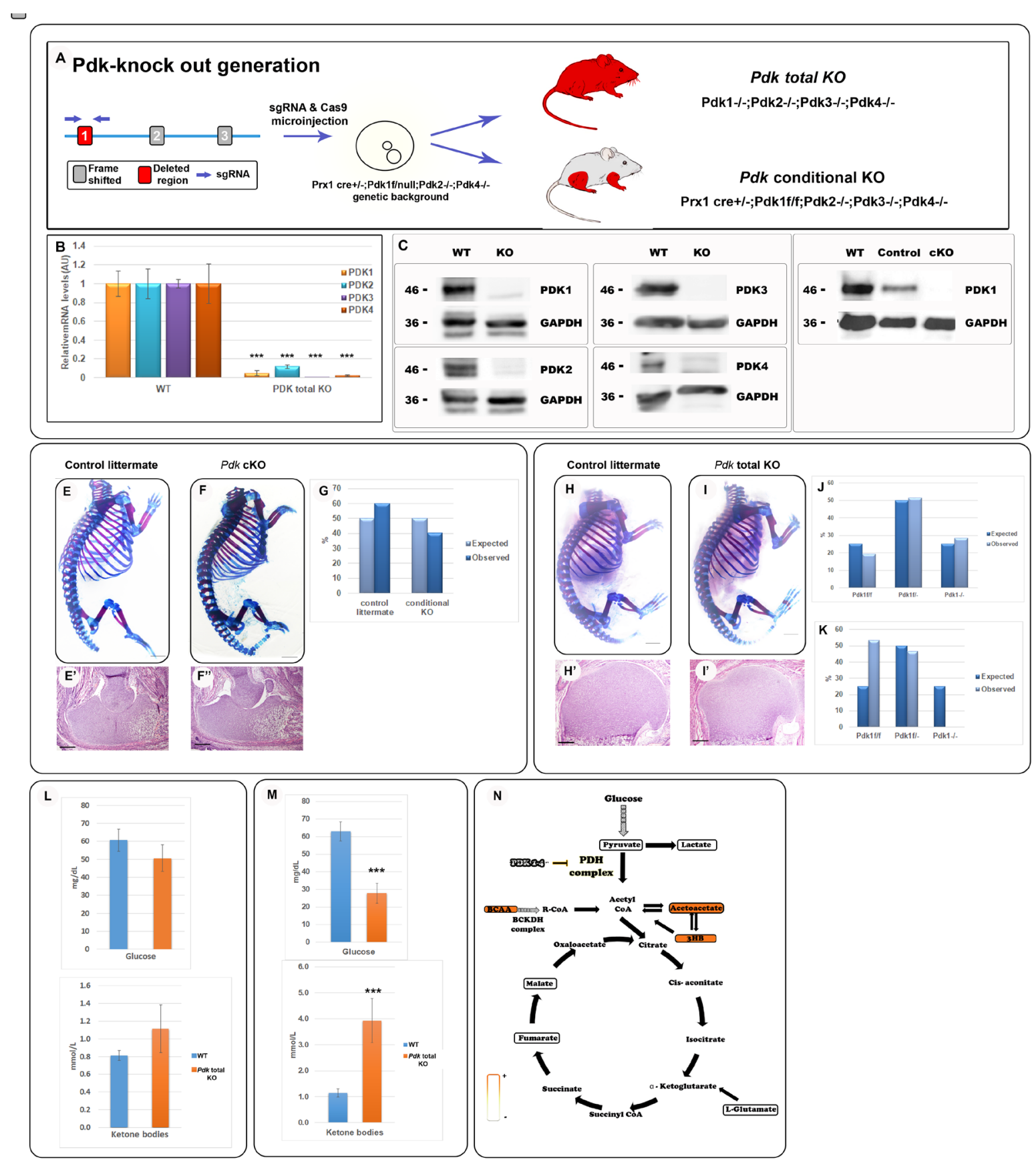

Figure 2. Deletion of the entire $\boldsymbol{P} \boldsymbol{d} \boldsymbol{k}$ family results in postnatal lethality. (A) Schematic representation of $P d k 3$ gene and the targeted site of CRISPR/Cas9-mediated deletion. This was followed by zygote injection to generate $P d k$ total KO mice or $P d k$ conditional KO in limb mesenchyme only, depending on the genetic background. (B) qRT-PCR of Pdk1-4 mRNA confirms deletion of all four genes, as compare to WT (***, $\mathrm{P}<0.0001 ; \mathrm{n}=4$ for each genotype; data are normalized to $T b p$ and presented as mean $\pm \mathrm{SD}$ ). (C) Protein expression analysis (western blot) of four PDK isoenzymes in heart samples derived from Pdk total KO or WT P0 pups shows complete deletion of the entire PDK family (representative images, $n=3$ ). (D) Western blotting of chondrocytes from $P d k \mathrm{cKO}$, control littermate and WT embryos shows deletion of PDK1 in the mutant (representative images, 3 biological repeats, $\mathrm{n}=5$ for each sample). (E-F') Skeletal preparations (E,F) and H\&E-stained olecranon and distal humerus sections (E',F') from E18.5 Prx $1-C r e ; P d k k^{f f f} ; P d k 2^{-/} ; P d k 3^{-/-}$ $; P d k 4^{-/}$embryos and control littermates (without Cre) show that the $P d k \mathrm{cKO}$ embryos develop normally (scale, $100 \mu \mathrm{m}$ ). (G) Graph showing Mendelian ratios of genotypes in $P d k$ cKO progeny. (H-I') Skeletal

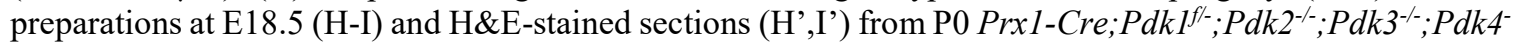


/ mice and control littermates (scale, $100 \mu \mathrm{m}$ ). (J) Graph showing Mendelian ratios of genotypes in E18.5 $P d k$ total KO embryos. (K) Graph showing non-Mendelian ratios of genotypes in $P d k$ total KO mature mice, as a result of loss of the total $\mathrm{KO}$ genotype. (L) Graphs showing in utero blood levels of glucose and 3HB, a ketone body, in $P d k$ total KO E18.5 embryos and P1 pups (M), compared to WT. Data are presented as mean \pm SE (embryos: $\mathrm{n}_{\mathrm{WT}}=9, \mathrm{n}_{\mathrm{KO}}=6 ; \mathrm{P} 1: \mathrm{n}_{\mathrm{WT}}=9 ; \mathrm{n}_{\mathrm{KO}}=4$, from 3 independent litters; $\mathrm{P}<0.05$, Student's $t$-test). (N) Scheme of serum metabolic profiles of P1 Pdk total KO pups, as determined by LC-MS polar metabolite analysis. Higher levels are shown in orange and lower levels in yellow, as compared to WT levels $(\mathrm{P}<0.05$; fold change $>2, \mathrm{n}_{\mathrm{WT}}=4 ; \mathrm{n}_{\mathrm{KO}}=3$ ). 


\section{PDC phosphorylation is maintained upon PDK family loss of function}

To gain molecular insight into the viability of $P d k$ total $\mathrm{KO}$ embryos, we studied the three phosphorylation sites on PDH1a subunit, which regulate PDC activity. First, we examined by western blot analysis PDC phosphorylation sites in chondrocytes isolated from growth plates of $P d k$ cKO embryos, which lack all $P d k$ genes in limb mesenchyme lineages. Interestingly, we found that one of the three sites, namely S300, was phosphorylated (Fig. 3A). To rule out the possibility that the phosphorylation of site S300 resulted from PDK1 contamination by surrounding tissues, such as skeletal muscles, we produced mouse embryonic fibroblasts (MEFs) from the total Pdk KO mice. As seen in Figure 3B, site S300 was phosphorylated in these MEFs as well. To strengthen the observation that the phosphorylation is maintained upon the loss of the $P d k$ family, we examined PDC phosphorylation in other tissues such as heart, lungs and kidney of $P d k$ total KO embryos. As seen in Figure S4, in all tested tissues, PDC was phosphorylated on at least one site in a tissue-specific manner. Altogether, these results suggest the existence of a backup mechanism that phosphorylates and inactivates PDC in the absence of all four PDKs. 


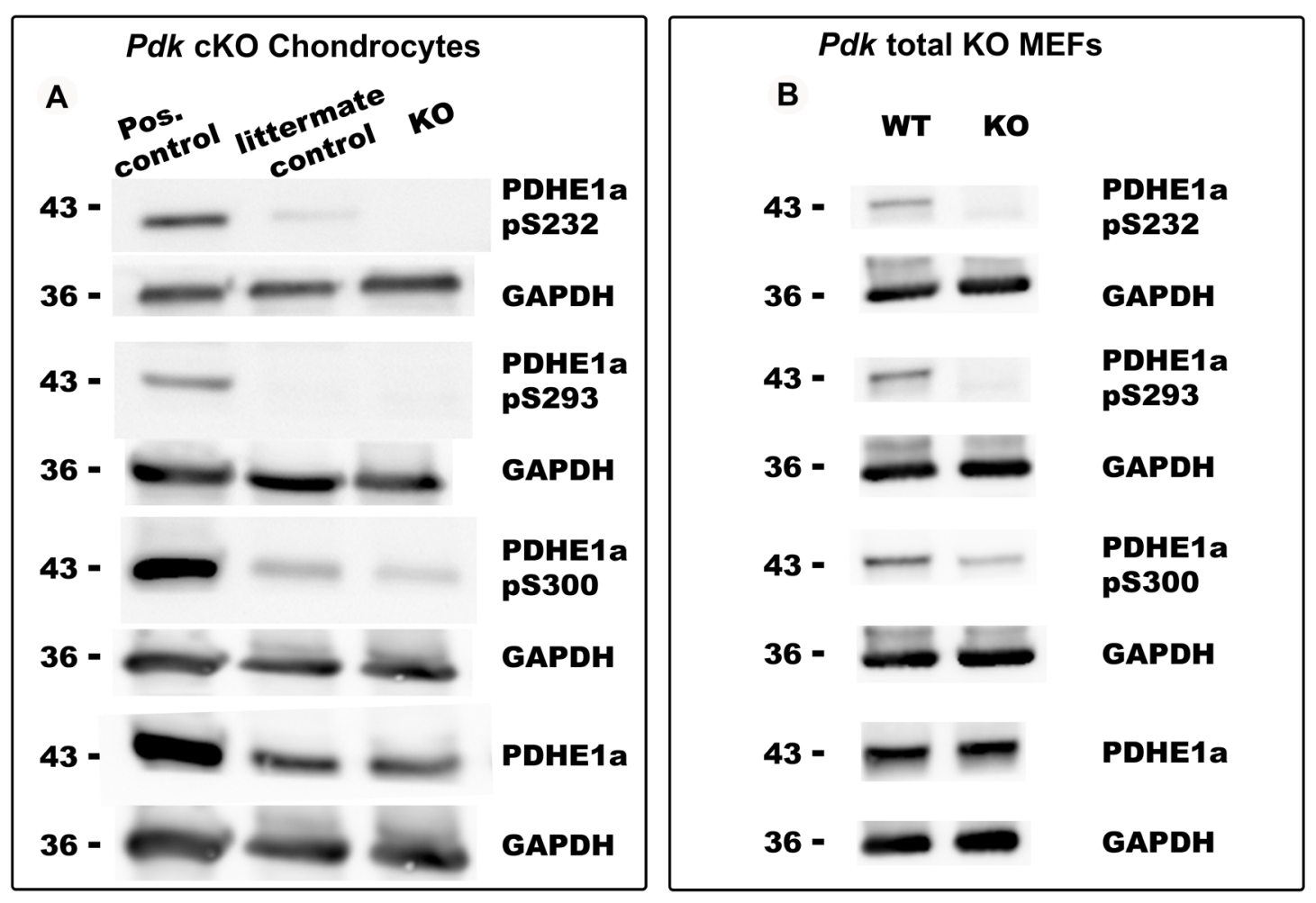

Figure 3. PDC phosphorylation is PDK-independent. Western blot analysis of the three PDC phosphorylation sites in chondrocytes extracted from growth plates of E17.5 Pdk cKO embryos (A; Prxl-

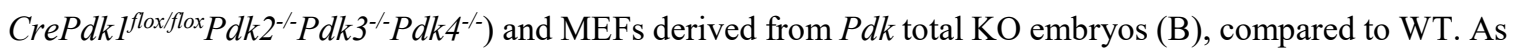
controls, PDH1a and endogenous GAPDH total protein levels were measured. Each chondrocyte sample is a pool of 5 biological repeats; $\mathrm{n}=3$ for all groups. Hearts of E17.5 WT embryos are shown as a positive control, littermate control were Prxl-Cre-negative. MEF samples, $\mathrm{n}=7$.

\section{$B c k d k$ is necessary for embryonic development in the absence of $\boldsymbol{P} d \boldsymbol{k}$ family}

Our results suggested that another kinase, which is not a PDK, phosphorylates PDH1a subunit of PDC. To identify this kinase, we searched for suitable candidates using bioinformatic tools, such as Ensemble ${ }^{38}$ and GeneCards ${ }^{39}$. One candidate that emerged was branched chain ketoacid dehydrogenase kinase $(B c k d k)$, which catalyzes the phosphorylation and inactivation of the branched-chain alpha-ketoacid dehydrogenase complex (BCKDC), the key regulatory enzyme of the valine, leucine and isoleucine catabolic pathways $^{40}$. GenesLikeMe analysis ${ }^{41}$ predicted that $B c k d k$ was a paralog of $P d k 1$ and belonged to the same mitochondrial kinase proteins family, mainly by sequence and 
domain similarity (Fig. 4A). Moreover, protein-protein interaction analysis by STRING ${ }^{42}$ predicted BCKDK to interact with PDH1a subunit of PDC (Fig. 4B).

Based on these observations, we hypothesized that BCKDK serves as the backup mechanism for PDK family during embryonic development by regulating PDC phosphorylation to inhibit its activity. To test this hypothesis, we sought to delete $B c k d k$ on Pdk family $\mathrm{KO}$ genetic background. $B c k d k \mathrm{KO}$ mice were previously reported to be viable at neonatal stages and to display growth retardation only at three weeks of age ${ }^{43}$. To avoid embryonic lethality, we opted to delete $B c k d k$ on the background of $P d k 1 \mathrm{cKO}$ combined with Pdk2-4 KO. Thus, we used the CRISPR/Cas9 method to generate a Prx1-

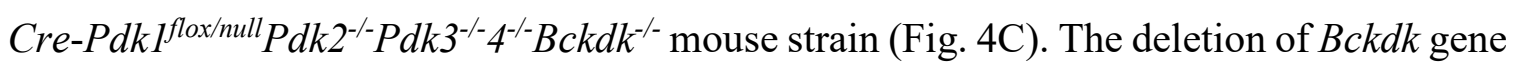
was verified at both mRNA and protein levels by qRT-PCR and western blotting, respectively (Fig. 4D,E). Crossing Prxl-Cre-Pdk1 flox/null $P d k 2^{-/} P d k 3^{-/-} 4^{-/-} B c k d k^{+/-}$to

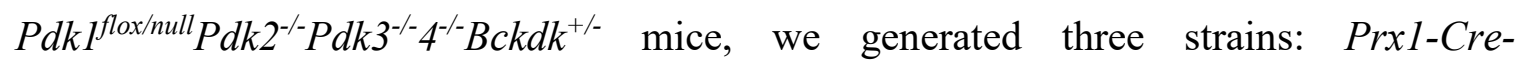

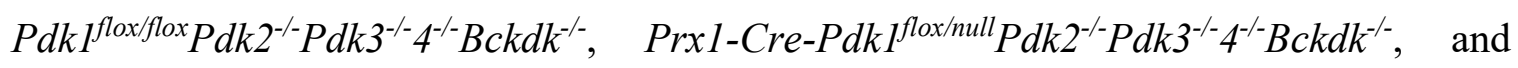

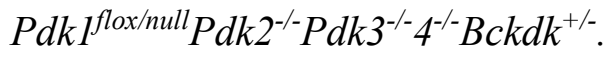

First, we examined the viability of embryos lacking $P d k 1$ in limb mesenchyme lineages and null for Pdk2,Pdk3,Pdk4 and Bckdk. For that, we crossed Prx1-Cre-Pdk floxflox Pdk2-/$P d k 3^{-/-} 4^{-/} B c k d k^{+/-}$mice with $P d k 1^{f l o x f l o x} P d k 2^{-/} P d k 3^{-/-} 4^{-/} B c k d k^{+/-}$and analyzed genotype distribution between E10.5 and E12.5, a stage at which Prx1-Cre is activated ${ }^{44}$ (Fig. 4F). Results showed that at E10.5 and E11.5, the numbers of Prx1-Cre-Pdk1 floxflox Pdk2-/-Pdk3${ }^{-} 4^{-/} B c k d k^{-/}$embryos were close to the expected Mendelian ratios. Yet, some of these embryos displayed developmental retardation in comparison to control littermates (Fig. $4 \mathrm{G})$. By contrast, at E12.5 the prevalence of this genetic combination was lower than expected, indicating embryonic lethality (Fig. 4F). Moreover, we failed to observe this combination postnatally (Fig. 4H).

To study genetic interaction between $P d k 1$ and $B c k d k$, we crossed $P d k 1^{f l o x / m u l l} P d k 2^{-/} P d k 3^{-}$

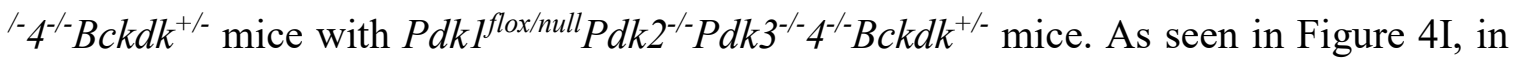
the presence of an intact $P d k l$ gene, all three combinations of $B c k d k$, namely WT, heterozygous and null, were observed. In contrast, heterozygosity of both Pdkl and Bckdk 
resulted in 50\% reduction from the expected ratio, and no $P d k 1^{\text {flox/null }} P d k 2^{-/-} P d k 3^{-/} 4^{-/-}$ $B c k d k^{-/-}$offspring were observed, suggesting genetic interaction between these genes.

Together, these results clearly suggest that loss of Bckdk on Pdk family KO background results in embryonic lethality and strongly support the hypothesis that BCKDK compensates for the absence of PDK isoenzymes during development.
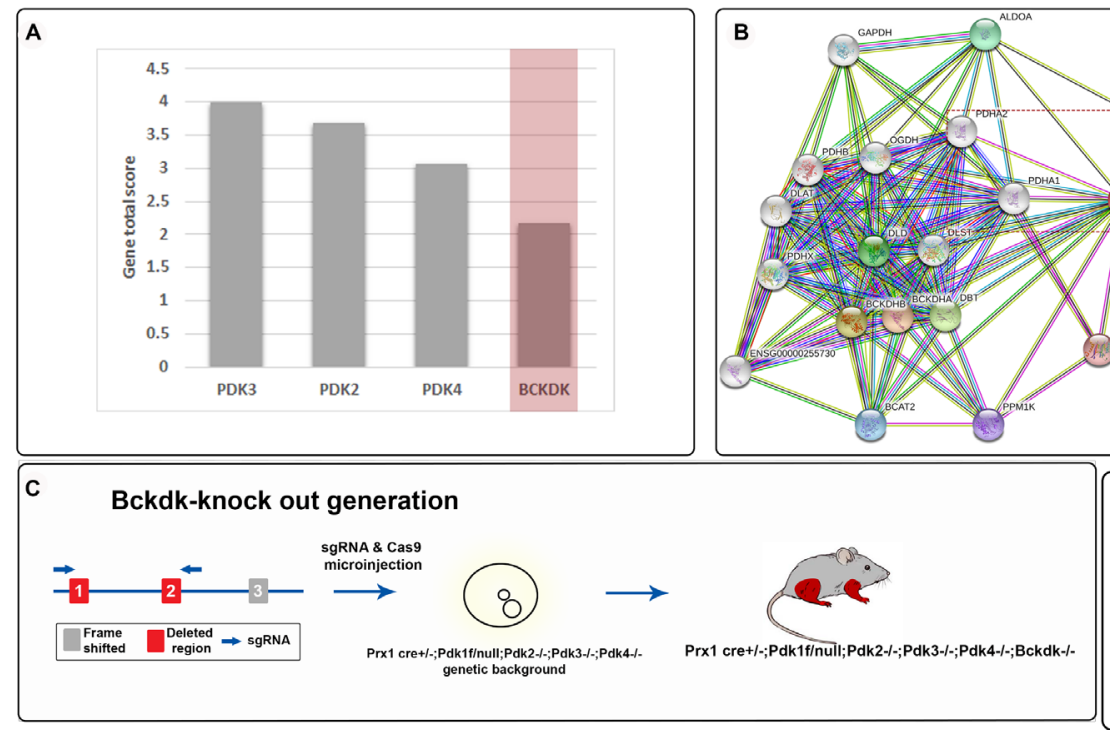

\begin{tabular}{|c|c|c|c|c|}
\hline \multicolumn{5}{|c|}{ F Prx1 cre+:Pdk1f/f;2;3;4KO;Bck+l- x Pdk1fff;2;3;4KO;Bck+/- } \\
\hline Embryonic day & \multicolumn{3}{|c|}{ e10.5 } & \\
\hline Genotype & Expected (\%) & \multicolumn{2}{|c|}{ Observed (\%) } & Expected ( \\
\hline Pdk1f/f;2;3;4KO;Bck WT & $5.3(12.5 \%)$ & \multicolumn{2}{|c|}{$6(14.6 \%)$} & $3.9(12.5 \%$ \\
\hline Pdk1f/f;2;3;4KO;Bck het & $10.5(25 \%)$ & & $7.8(25 \%)$ \\
\hline Pdk1f/f;2;3;4KO;BCk KO & $5.3(12.5 \%)$ & \multicolumn{2}{|c|}{$3(7.3 \%)$} & $3.9(12.5 \%$ \\
\hline Prx1 cre:Pdk1f/f;2;3;4KO;Bck WT & $5.3(12.5 \%)$ & \multicolumn{2}{|c|}{$5(12.2 \%)$} & $3.9(12.5 \%$ \\
\hline Prx1 cre:Pdk1f/f;2;3;4KO;Bck het & $10.5(25 \%)$ & \multicolumn{2}{|c|}{$11(26.8 \%)$} & $7.8(25 \%)$ \\
\hline Pr×1 cre:Pdk1f/f;2;3;4KO;Bck KO & $5.2(12.5 \%)$ & \multicolumn{2}{|c|}{$4(9.8 \%)$} & $3.9(12.5 \%$ \\
\hline Total & \multirow{2}{*}{\multicolumn{3}{|c|}{$\begin{array}{r}n=41 \\
0.890 \\
\end{array}$}} & \\
\hline Chi-Squaretest & & & & \\
\hline \multicolumn{5}{|c|}{ H Prx1 cre+:Pdk1fff;2;3;4KO;Bck+/- x Pdk1ff; 2;3;4KO;Bck -/- } \\
\hline Genotype & \multicolumn{4}{|c|}{ Expected (\%) Observed (\%) } \\
\hline Pdk1f/f;2;3;4;Bck het & \multicolumn{2}{|c|}{$4.8(25 \%)$} & \multicolumn{2}{|c|}{$12(46 \%)$} \\
\hline Pdk1f/f;2;3;4;Bck KO & \multicolumn{2}{|c|}{$4.8(25 \%)$} & \multicolumn{2}{|c|}{$9(35 \%)$} \\
\hline Prx1 cre;Pdk1f/f;2;3;4;Bck het & \multicolumn{2}{|c|}{$4.8(25 \%)$} & \multicolumn{2}{|c|}{$5(19 \%)$} \\
\hline Prх1 cre;Pdk1f/f;2;3;4;Bck КО & & $25 \%)$ & & $\%)$ \\
\hline Total & \multicolumn{4}{|c|}{$\mathrm{N}=26$} \\
\hline Chi-Square test & \multicolumn{4}{|c|}{$2.02 \mathrm{E}-04$} \\
\hline
\end{tabular}

\begin{tabular}{cc|cc}
\multicolumn{2}{c}{ e11.5 } & \multicolumn{2}{c}{ e12.5 } \\
$(\%)$ & Observed (\%) & Expected (\%) & Observed (\%) \\
$(5 \%)$ & $2(6.5 \%)$ & $13.3(12.5 \%)$ & $22(20.8 \%)$ \\
$\%)$ & $7(22.6 \%)$ & $26.5(25 \%)$ & $34(32.1 \%)$ \\
$5 \%)$ & $10(32.3 \%)$ & $13.3(12.5 \%)$ & $15(14.2 \%)$ \\
$5 \%)$ & $2(6.5 \%)$ & $13.3(12.5 \%)$ & $7(6.6 \%)$ \\
$\%)$ & $7(22.6 \%)$ & $26.5(25 \%)$ & $24(22.6 \%)$ \\
$\%)$ & $3(9.7 \%)$ & $13.3(12.5 \%)$ & $4(3.8 \%)$ \\
$n=31$ & \multicolumn{2}{c}{$n=106$} \\
0.037 & \multicolumn{2}{c}{$6.02 E-11$} \\
\hline
\end{tabular}
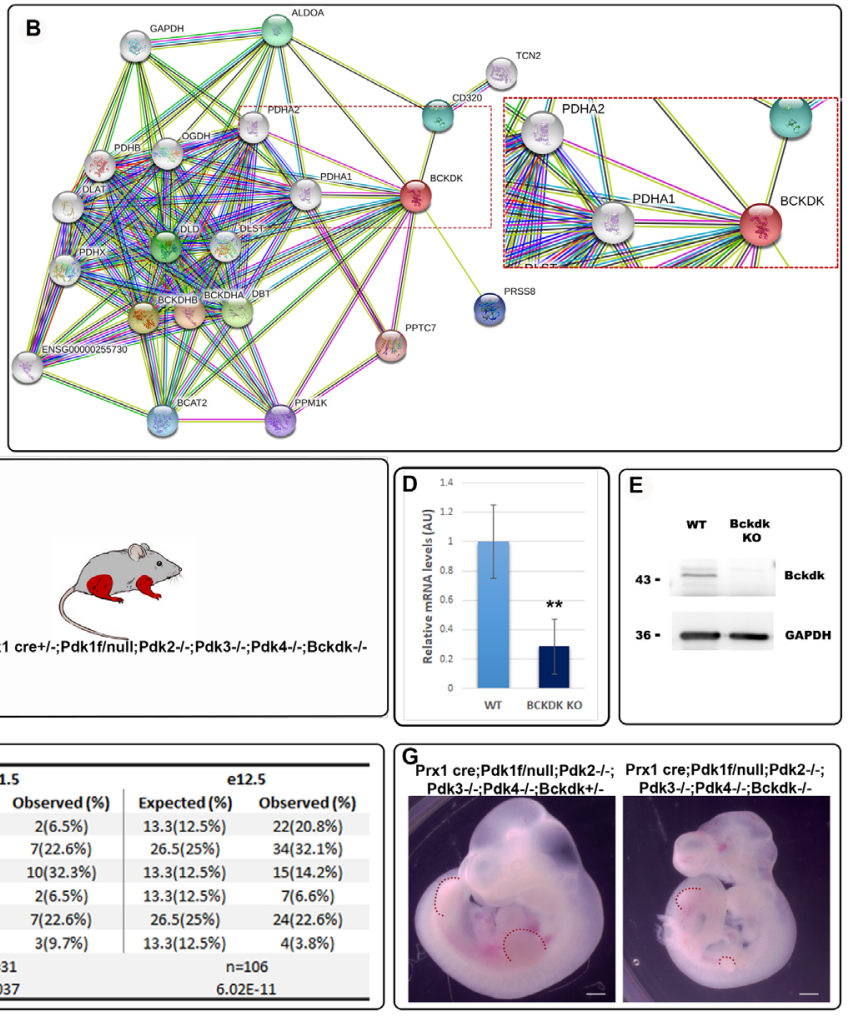

\begin{tabular}{|c|c|c|}
\hline \multicolumn{3}{|c|}{ I Pdk1fff;2;3;4KO;Bck+/- x Pdk1f/null;2;3;4KO;Bck+/- } \\
\hline Genotype & \multicolumn{2}{|c|}{ Expected (\%) Observed (\%) } \\
\hline Pdk1f/f;2;3;4KO;Bck WT & $5.1(12.5 \%)$ & $10(24.4 \%)$ \\
\hline Pdk1f/f;2;3;4KO;Bck het & $10.3(25 \%)$ & $12(29.3 \%)$ \\
\hline Pdk1f/f;2;3;4KO;Bck KO & $5.1(12.5 \%)$ & $3(7.3 \%)$ \\
\hline Pdk1f/null;2;;3;4KO;Bck WT & $5.1(12.5 \%)$ & $11(26.8 \%)$ \\
\hline Pdk1f/null;2;;;AKO;Bck het & $10.3(25 \%)$ & $5(12.2 \%)$ \\
\hline Pdk1f/null;2;;3;4KO;BckKO & $5.1(12.5 \%)$ & $0(0 \%)$ \\
\hline Total & \multicolumn{2}{|c|}{$n=41$} \\
\hline Chi-Squaretest & \multicolumn{2}{|c|}{0.001} \\
\hline
\end{tabular}

Figure 4. Prxl-CrePdk cKO-Bckdk KO mice display embryonic lethality. (A) GenesLikeMe analysis identifies $B c k d k$ as a $P d k 1$ paralog, along with the other $P d k$ family members (total score combines sequences, domains, super pathways, expression, compounds and gene ontology similarities). (B) STRING analysis predicts protein-protein interaction between BCKDK and PDH complex subunit PDH1a (string 
legend: yellow, textmining; black, co-expression; pink, experimentally determined). (C) Schematic representation of $B c k d k$ gene and the targeted sites of CRISPR/Cas9-mediated deletion. This was followed by zygote injection to generate Prx $1-C r e-P d k 1^{f l o x / n u l l} P d k 2^{-/-} P d k 3^{-/-} 4^{-/-} B c k d k^{-/-}$mouse, lacking $P d k 1$ in limb mesenchyme lineages. (D) qRT-PCR of Bckdk mRNA in Prx1-Cre-Pdk $1^{\text {flox } / n u l l} P d k 2^{-/-} P d k 3^{-/-} 4^{-/-}$ $B c k d k^{-/-}$mice, as compare to WT (**, $\mathrm{P}<0.001 ; \mathrm{n}=3$ for each genotype; data normalized to $T b p$ and presented as mean $\pm \mathrm{SD}$ ). (E) Protein expression analysis by western blotting shows complete deletion of BCKDK in $P d k 1^{\text {floxflox }} P d k 2^{-/-} P d k 3^{-/-} 4^{-/-} B c k d k^{-/-}$MEF cells $(\mathrm{n}=3)$. (F) Expected Mendelian ratios and observed genotype distribution of Prx1-Cre-Pdk1 floxflox $P d k 2^{-/-} P d k 3^{-/-} 4^{-/-} B c k d k^{+/-}$crossed with $P d k 1^{\text {floxflox }} P d k 2^{-/-}$ $P d k 3^{-/-} 4^{-/-} B c k d k^{+/-}$embryos at E10.5 (n=41), E11.5 (n=31) and E12.5 (n=106). (G) Representative E10.5 Prx 1-Cre-Pdk1 floxflox $P d k 2^{-/-} P d k 3^{-/-} 4^{-/-} B c k d k^{-/-}$embryo displays delayed development as compared to control Bckdk heterozygous littermate (dashed red lines indicate the limbs; scale, $50 \mu \mathrm{m})$. (H) Mendelian ratios and genotype distributions of Prxl-Cre-Pdk $1^{f l o x f l o x} P d k 2^{-/-} P d k 3^{-/-} 4^{-/} B c k d k^{+/-}$crossed with

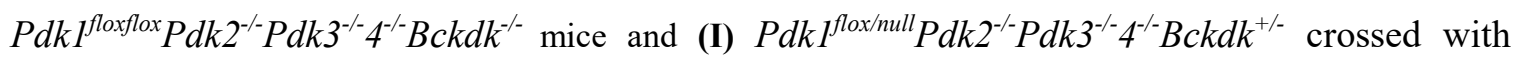

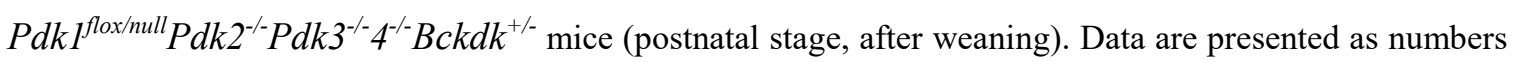
and percentage of the total progeny, statistical significance was determined by Chi-square test.

\section{BCKDK regulates PDC phosphorylation and activity as well as pyruvate flux into the}

\section{TCA cycle}

To further validate our hypothesis, we proceeded to test directly if $B c k d k$ is necessary for the phosphorylation of PDC subunit PDH1a in the absence of all $P d k$ family members. To overcome the early embryonic lethality of mice lacking all $P d k$ genes and $B c k d k$, we used

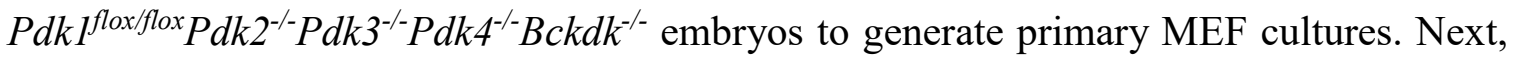
we infected these cells with adeno-Cre virus to ablate the expression of $P d k 1$, or with adeno-GFP as a control. As seen in Figure 5A, upon infection with adeno-Cre, 
concomitantly with the reduction in PDK1 expression, PDH1a phosphorylation on site S300 was reduced as compared to control cells; however, some phosphorylation was still noticeable. Since we observed low levels of PDK1 expression in adeno-Cre infected cells, we inhibit the activity of the remaining PDK1 by supplementing the culture with dichloroacetate (DCA), a PDK inhibitor ${ }^{45}$. As seen in Figure 5A, DCA nearly eliminated the phosphorylation of site S300. These results provide strong molecular evidence to the ability of BCKDK to regulate PDC by phosphorylation.

To demonstrate the biochemical effect of this regulation, we next quantified PDC activity in cells lacking $P d k$ family and in cells lacking both $P d k$ family and $B c k d k$. First, we examined PDC activity in MEFs from WT or $P d k$ total $\mathrm{KO}$ embryos. As seen in Fig. 5B, $P d k$ family loss resulted, as expected, in a significant two-fold increase in PDC activity, as compared to WT cells. Next, we examined PDC activity in our primary $P d k 1^{f l o x f f l o x} P d k 2^{-/-}$ $P d k 3^{-/} P d k 4^{-/} B c k d k^{-/-}$MEFs, which also lack Bckdk. Whereas in control MEFs infected with adeno-GFP virus ( $P d k \mathrm{cKO}-B c k d k \mathrm{KO}$ GFP), which retain $P d k 1$ expression, the increase in PDC activity was similar to the one observed in Pdk total $\mathrm{KO}$ cells, infection with adeno-Cre virus ( $P d k \mathrm{cKO}-B c k d k \mathrm{KO} \mathrm{CRE})$ further increased the signal by 1.5 times relative to adeno-GFP infected MEFs and $P d k$ total KO-derived MEFs, and by 3 times relative to the WT.

Next, to provide direct metabolic evidence to the consequences of the observed changes in PDC activity, we examined pyruvate flux into the TCA cycle in these cell lines by performing ${ }^{13} \mathrm{C}$ tracer experiments. Using labeled $\left[3-{ }^{13} \mathrm{C}\right]$ pyruvate, we measured the fraction of $\mathrm{m}+2$ mass isotopomers of TCA cycle intermediates, namely citrate, $\alpha-$ ketoglutarate, succinate and fumarate, from the total abundance of these intermediates. As seen in Figure 5C, the fractions of labeled intermediates increased significantly in $P d k$ total 
KO MEFs relative to WT cells. Predictably, upon deletion of $P d k$ family and $B c k d k$ ( $P d k$ cKO-Bckdk KO CRE), the fractions of these metabolites further increased, as compared to both GFP control (Pdk cKO-Bckdk KO GFP) and $P d k$ total KO MEFs.

Collectively, these results provide strong molecular and biochemical evidence to the ability of BCKDK to regulate PDC phosphorylation and, thereby, activity.
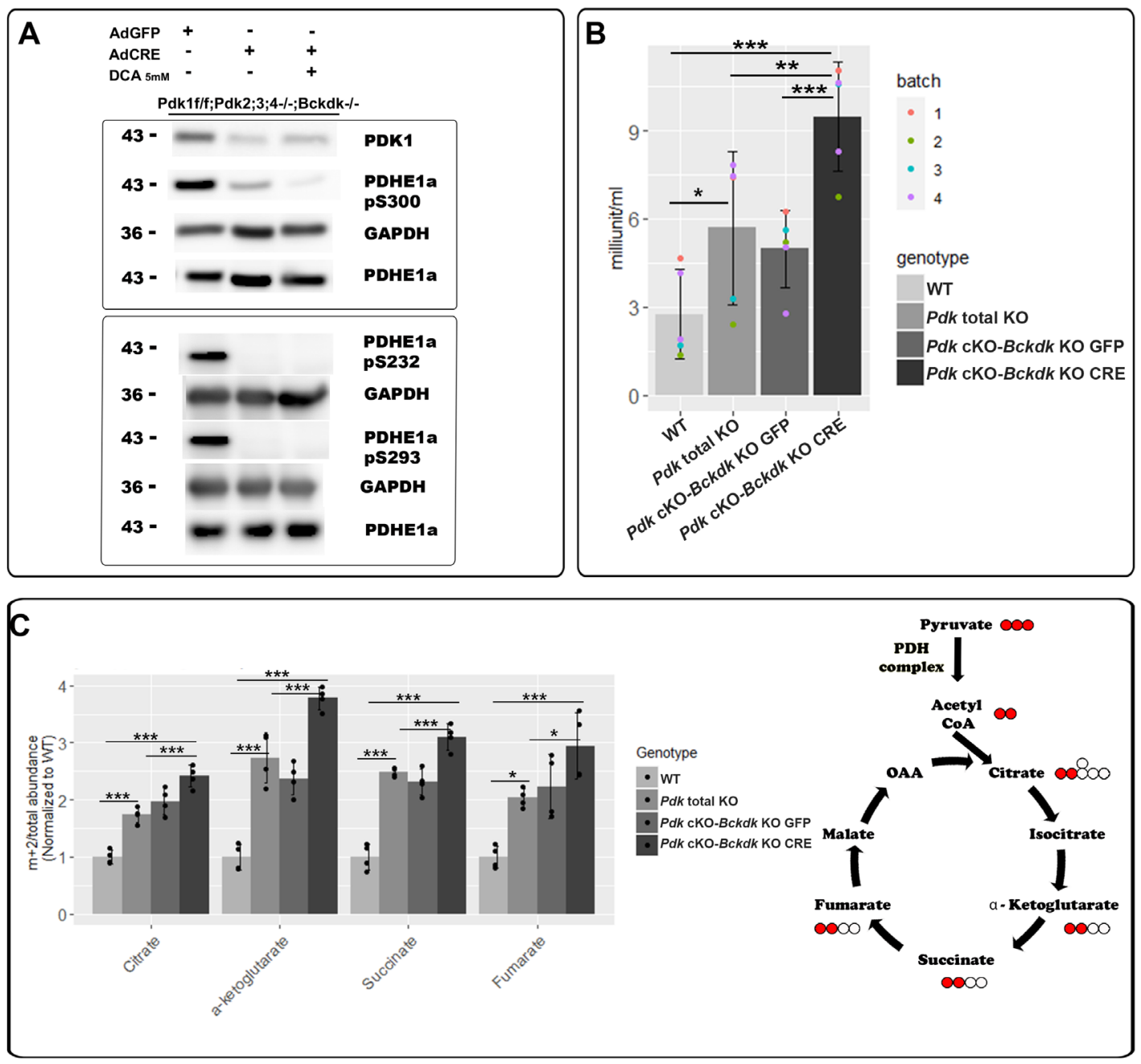

Figure 5. PDC phosphorylation and activity is BCKDK-dependent. (A) Western blot of PDK1 and of PDH1a phosphorylated sites pS300, the main active PDC site in MEFs, pS232 and pS293 in MEFs derived from $P d k 1^{\text {floxfllox }} P d k 2^{-/} P d k 3^{--} P d k 4^{-/} B c k d k^{-/}$mice infected with either Adeno-GFP as a control, or Adeno-CRE with or without addition of $5 \mathrm{mM}$ DCA, to ablate PDK1 (representative images, $n=6$ from two different sets of experiments). (B) PDC activity assay in MEFs from $P d k$ 
total KO or $P d k 1^{f l o x f f l o x} P d k 2^{-/} P d k 3^{-/} P d k 4^{-/} B c k d k^{-/}$mice infected with either adeno-GFP ( $P d k \mathrm{cKO}-$ $B c k d k$ KO GFP) or adeno-Cre ( $P d k \mathrm{cKO}-B c k d k \mathrm{KO} \mathrm{CRE})$, and WT as a control (mean $\pm \mathrm{SD}$, *** $\mathrm{P}<0.001, * * \mathrm{P}<0.01, * \mathrm{P}<0.05, \mathrm{n}=5$ for each genotype from 4 different experiments, ANOVA multiple comparison test). (C) Analysis of $\left[3-{ }^{13} \mathrm{C}\right]$ pyruvate flux into the TCA cycle using

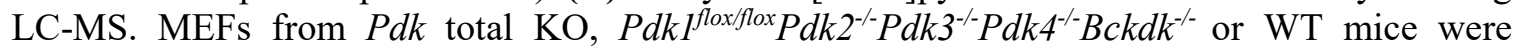
infected with either adeno-GFP ( $P d k \mathrm{cKO}-B c k d k \mathrm{KO}$ GFP) or adeno-Cre ( $P d k \mathrm{cKO}-B c k d k \mathrm{KO}$ $\mathrm{CRE})$. Graph shows the fraction of $\mathrm{m}+2$ mass isotopomers of TCA cycle intermediates from the total abundance of these metabolites, normalized to WT levels. On the right is a schematics of the TCA cycle showing the labeled metabolites (mean $\pm \mathrm{SD}, * * * \mathrm{P}<0.001, * * \mathrm{P}<0.01, * \mathrm{P}<0.05$, $\mathrm{n}=4$ for each genotype, ANOVA multiple comparison test). 


\section{DISCUSSION}

The ability of cells to adapt to different environmental conditions and maintain energetic homeostasis is one of the hallmarks of life. Using oxygen as the most common electron acceptor allows cells to maximize utilization of acetyl CoA driven from three catabolic pathways of glucose, amino acids and fatty acids feeding the TCA cycle. In this work, we discover a novel regulatory metabolic circuit between glucose and BCAA catabolic pathway. We found that BCKDK, a key regulator of BCAA catabolic pathway, regulates the activity of PDC, the gatekeeper of the glucose catabolic pathway. As the underlying mechanism, we show that BCKDK regulates PDC phosphorylation. This finding establish BCKDK as a backup mechanism for the PDK family in regulating PDC activity, which allows cellular metabolic balance to sustain embryonic development (Fig. 6).

BCAAs are a group of indispensable amino acids that includes leucine, isoleucine, and valine $^{43}$. Their catabolism serves the cellular energetic balance by feeding the TCA cycle through a two-step degradation pathway of transamination to form branched chain $\alpha$-keto acids (BCKAs), followed by oxidation and decarboxylation resulting in an irreversible synthesis of acetyl-CoA entering the TCA cycle ${ }^{46}$. The second step is crucial for degradation by $\mathrm{BCKDC}$ multienzyme. Therefore, BCKDC regulation is important to maintain proper levels of BCAAs and normal development ${ }^{43}$. Interestingly, PDC and BCKDC share many structural and enzymatic properties, as well as mechanism of regulation. Similarly to $\mathrm{PDC}, \mathrm{BCKDC}$ is regulated by reversible phosphorylation of its $\mathrm{E} 1$ subunit on two serine sites (ser293 and ser303) mediated by its own native kinase, BCKDK. The activity of the entire BCKDC complex depends on BCKDK function, which is regulated by a feedback loop of substrate metabolites, e.g. BCAA blood levels ${ }^{47}$. 
Over the years, an interplay between the glucose and BCAA catabolic pathways has been established ${ }^{46}$. Recently, it was shown that these pathways can regulate one another through feedback loops of nutrients blood-circulation, i.e, glucose and amino acids, to maintain energetic balance. Excessive or insufficient levels of glucose or BCAAs was shown to influence transcription of elements of the parallel pathway. For example, BCAA levels can improve glucose uptake by upregulation of glucose transporters ${ }^{48}$, and a reciprocal effect was shown when high levels of glucose suppresses the expression of BCAA degradation enzymes. ${ }^{49}$. Our discovery that BCKDK can phosphorylate PDC uncovers a new level of regulation that hardwires glucose and BCAA degradation pathways through direct enzymatic regulation and not by a feedback loop of circulating nutrients.

From an evolutionary viewpoint, Oppenheim et. al ${ }^{50}$ recently showed that in some parasites lacking mitochondrial PDC, acetyl-CoA is converted from pyruvate by BCKDC, which fulfills PDC function. Therein, it was suggested that BCKDC evolved earlier than PDC. Although the ancestral gene of the regulators of these complexes, i.e. $P d k$ and $B c k d k$, is yet to be discovered, sequence similarities between $P d k$ and $B c k d k$ has placed them as paralogs belonging to the same mitochondrial kinase family ${ }^{51-54}$. Here, we provide strong in vivo evidence suggesting that $B c k d k$ is not only a paralog, but also a functional paralog of the $P d k$ family. One interesting question that remains is whether BCKDK acts only as a backup mechanism for the loss of the PDK family, or is it a part of PDC regulation in normal conditions as well. Another intriguing question that is raised by the observed functional compensation is whether PDKs can compensate for the absence of BCKDK. This assumption makes sense considering that $B c k d k \mathrm{KO}$ mice are viable and exhibit growth retardation only at three weeks of age, due to lack of BCKDC regulation ${ }^{43}$. 


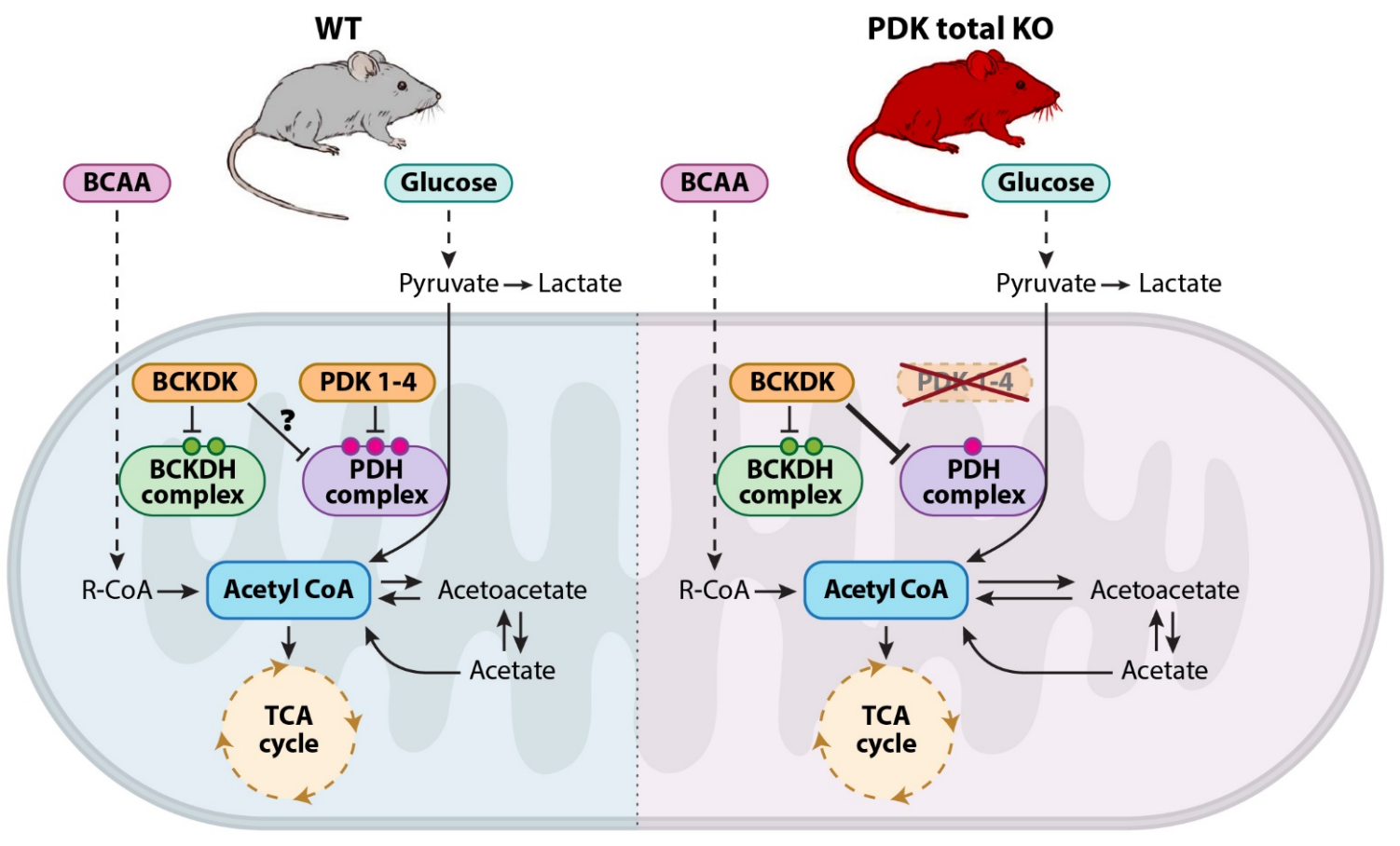

Figure 6. BCKDK compensates for Pdk loss of function via PDC phosphorylation.

Cartoon depicting the interplay between glucose and BCAA catabolism in WT (left) versus $P d k$ total $\mathrm{KO}$ mice (right). In WT mice, PDC is inactivated by phosphorylation on three serine residues, whereas in the $P d k$ total $\mathrm{KO}$ mice, these phosphorylation sites are $B c k d k$ dependent. This novel level of regulation implicates BCKDK as a functional paralog of the PDK family, which may also act in the same way in the presence of PDKs.

Another interesting point emerging from our finding that BCKDK can regulate PDC is the tissue-specific expression of PDK isoenzymes ${ }^{52}$. Moreover, each isoenzyme was shown to be regulated by different physiological conditions ${ }^{5,9,10}$. By contrast, several studies support the possibility of functional redundancy among PDK family members, for example, between PDK1 and PDK2 in muscle tissue in vitro ${ }^{31}$ and between PDK2 and PDK4 in vivo $^{33}$. Our failure to identify major developmental phenotype in embryos that lost different $P d k$ genes increases the scope of known functional redundancy among these four isoenzymes. The sequential targeting of these genes demonstrates that each PDK can 
compensate for the loss of the other family members. Another level of this complex regulation relates to the tightly controlled rate of PDC phosphorylation, which is maintained by the balance between the activity of PDK and pyruvate dehydrogenase phosphatases (PDPs). The latter are two enzymes that reverse PDK activity and thereby reactivate $\mathrm{PDC}^{55}$. Given the importance of this balance, it is interesting to see whether the activity levels of PDKs and PDPs are regulated such that a reduction in PDK activity would lead to a concomitant reduction in PDP activity. Such coordination might contribute to the maintenance of PDC phosphorylation in the absence of PDK family. Overall, PDC regulation by kinase versus phosphatase activity should be further studied under different physiological conditions.

On that note, hypoxic conditions have long been associated with pathologies, such as tissue ischemia, inflammation and cancer ${ }^{56}$. However, hypoxic microenvironments were shown to be essential for mammalian embryonic development ${ }^{24}$. In either state, HIF1 transcription factor initiates a key molecular response that maintains $\mathrm{O}_{2}$ homeostasis and metabolic balance $^{14,22}$. Specifically, the HIF1 $\alpha$-PDK axis is considered a major regulator of cell adaptation to hypoxia by both controlling energy production and decreasing mitochondrial oxygen consumption ${ }^{12-14,16,18,57}$. However, our results of normal development in the absence of one side of this axis, namely all PDKs, question its significance. There are three possibilities to reconcile our findings with the common view. One is that HIF1 $\alpha$ activation of a wide range of downstream target genes is adaptive enough to support metabolic energy balance under hypoxia even without inactivation of PDC. For example, by activating pyruvate kinase isoform M2 (PKM2), which diverts pyruvate to the pentose phosphate pathway ${ }^{18}$. Second, it is possible that by activating another, yet undescribed mechanism, HIF $1 \alpha$ compensates for the absence of PDK family. In that context, the possibility that 
HIF $1 \alpha$ can regulate $B c k d k$ should be considered. Finally, it is possible that the involvement of HIF $1 \alpha$-PDK axis is restricted to the development or function of specific organs, which are dispensable for embryonic development. In support of this explanation is our finding that mice lacking all $P d k$ genes in limb mesenchyme developed normally, whereas total Pdk KO mice died at early neonatal stages. The metabolic abnormalities in these newborn total $\mathrm{KO}$ mice strongly imply that the affected organs are involved in physiological regulation of the organism, which is critical for its survival postnatally but not during embryogenesis. It is also possible that during development, the consequences of metabolic abnormalities, such as ketone body accumulation and hypoglycemia, are buffered by the safe environment of the placenta ${ }^{58}$. Obviously, the different outcomes of embryonic development and postnatal growth of $P d k$ total $\mathrm{KO}$ mice does not exclude the possibility that these two processes have different metabolic requirements. In that case, it is possible that BCKDK could backup for PDKs only during the embryonic period.

To conclude, our findings provide new insight into the functional redundancy among PDK family members in modulating PDC activity to maintain energy production and cell survival, while revealing the existence of a previously unknown backup mechanism, which places $B c k d k$ as a functional paralog of $P d k$. This backup mechanism unveils a new level of regulatory crosstalk between two central metabolic pathways that feed the TCA cycle. The finding of this backup mechanism may promote the development of new therapeutic strategies for complex diseases involving changes in the metabolic state of cells, such as cancer, diabetes and many other metabolic diseases. 


\section{Author contribution}

Experiment Design, L.H-Y, L.B, and E.Z; Experiments, L.H-Y, L.B, N.F, R-C.V, N.M, S.K, A.S, M.C, S.B-D.O.B, R.H-K, M.I and S.M; Intellectual Contributions, E.Z, A.E, L.H-Y, L.B, N.F, R-C.V; Manuscript Writing, L.H-Y and E.Z with input from all authors.

\section{Acknowledgments}

We thank Nitzan Konstantin for expert editorial assistance, Dr. Robert Harris from Indiana University, who kindly provided us with PDK2 and PDK4 mice, Dr. Nicola Brunetti-Pierri from the Telethon Institute of Genetics and Medicine for his support and advice, Dr. Ron Rotkoff for his help with statistical analysis, Dr. Tsviya Olender for bioinformatics advice, and Neria Sharabi from the Department of Veterinary Resources for his help with mouse maintenance. Special thanks to all members of the Zelzer Laboratory for encouragement and advice. 


\section{STAR Methods}

\section{RESOURCE AVAILABILITY}

\section{Lead Contact}

Further information and requests for resources and reagents should be directed to and will be fulfilled by the Lead Contact, Elazar Zelzer (Eli.zelzer@weizmann.ac.il)

\section{Materials Availability}

All unique reagents generated in this study are available from the Lead Contact.

\section{Data and Code Availability}

The data reported in this study are available from the Lead Contact.

\section{EXPERIMENTAL MODEL AND SUBJECT DETAILS}

\section{Mouse lines}

Pdk2 KO (Vandenboom et al., 2011) and Pdk4 KO mice (Ho Jeoung et al., 2006) were previously described. The $P d k 1 \mathrm{KO}$, floxed-Pdkl and $P d k 1-l a c Z$ mice were generated as follows: Embryonic stem cells with a gene trap insertion (KO-first allele) in the Pdk1 gene were obtained from the European Conditional Mouse Mutagenesis program (EUCOMM) (Pettitt et al., 2013). Pdkl KO mice were crossed with PGK-Cre mice (Lallemand, Luria, Haffner-Krausz, \& Lonai, 1998) to generate the null Pdkl-lacZ mice. Alternatively, Pdk1 KO mice were crossed with Rosa26-FLPe mice (Jackson laboratory, 016226) to generate the floxed-Pdkl mice. Genotyping of Pdk1 KO, Pdkl-lacZ and floxed-Pdkl mice was performed by PCR (primer sequences are shown in Supplementary Table S1).

Generation of double and triple KO combinations of $P d k 1, P d k 2$ and $P d k 4$ was done by strain breeding. Pdk3 KO and Bckdk KO were generated as follows: Cas9 plasmid and plasmids encoding guide RNAs were designed and optimized for the best guides using several CRISPR designing tools, including the MIT CRISPR design tool (Hsu et al., 2013) and sgRNA Designer, Rule Sets 1 and 2 (Doench et al., 2016, 2014), in both the original sites and later in the Benchling implementations (www.benchling.com), SSC (Xu 
et al., 2015), and sgRNAscorer (Chari, Moosburner, \& Church, 2015), in their websites. The following oligos were used for construction of gRNA: Pdk3: 5'CGGGTtGGGGAGGTCTAGAG-3' (upstream of the putative TSS) and 5'ATTCCGTGAGAAGCTCCGGG-3' (downstream of the ATG in the first exon), total deletion of 560 bp (location X:93831603-93832603). For Bckdk: 5'CCCGCGCGATGTTACAGCCG-3' (an upstream guide around the TSS) and 5'CTCTACATGGTGTGTATCGG-3' (downstream of the ATG in the second exon), total deletion of 884bp (location chr7:127903907-127905206). In vitro transcribed Cas9 RNA (100 ng $\mathrm{ml} \mathrm{1)} \mathrm{and} \operatorname{sgRNA}(50 \mathrm{ng} \mathrm{ml} 1)$ were injected into one-cell stage fertilized embryos isolated from superovulated Prx1-Cre;Pdk $1^{f l o x /-} P d k 2^{-/-} P d k 4^{-/-}$or Prx1-Cre;Pdk $1^{f l o x /-} P d k 2^{-/-}$ $P d k 3^{-/-} P d k 4^{-/-}$mice mated with males of the same mutation to generate $P d k$ total $\mathrm{KO}$ or $P d k$ $\mathrm{cKO}-B c k d k \mathrm{KO}$ mice, respectively. Injected embryos were transferred into the oviducts of pseudopregnant ICR females as previously described (Jaenisch et al., 2013). Genomic DNA from treated embryos was analyzed by PCR primers designed to verify the deleted sequence of each gene; primer sequences are shown in Supplementary Table S1.

Staff and veterinary personnel monitored all mouse strains daily for health and activity. Mice were given ad libitum access to water and standard mouse chow with 12-hr light/dark cycles. All animal procedures were approved by the Institutional Animal Care and Use Committee and performed in strict adherence to Weizmann Institute Animal Care and Use guidelines, following the NIH, European Commission, and Israeli guidelines. In all timed pregnancies, plug date was defined as E0.5. For harvesting of embryos, timed-pregnant female mice were sacrificed by $\mathrm{CO}_{2}$ intoxication. The gravid uterus was dissected out and suspended in a bath of ice-cold PBS and the embryos were harvested. Tail genomic DNA was used for genotyping. All procedures and treatments are described as in Method Details. None of the mice was involved in any previous procedures prior to the study.

\section{Primary cultures}

For chondrocyte primary culture, hindlimb tibiofemoral growth plates of E17.5 Pdkl KO,

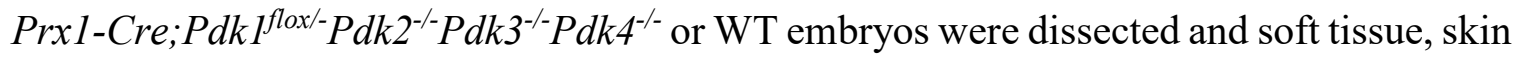
and particularly muscles were removed. The growth plates were dissected and placed in DMEM $4500 \mathrm{mg} / 1$ glucose (Thermofisher) with 1\% pen-strep solution (Biological 
Industries). Growth plates were digested in trypsin containing 0.25\% EDTA (Biological Industries) for 30 minutes at $37^{\circ} \mathrm{C}$ and in $1 \mathrm{mg} / \mathrm{ml}$ collagenase type $\mathrm{V}$ (Sigma) in DMEM $4500 \mathrm{mg} / \mathrm{l}$ glucose with $1 \%$ pen-strep solution for 2 hours. Chondrocytes were plated at a density of $125 \times 103$ cells $/ \mathrm{ml}$ and grown under normoxia in monolayer cultures in high glucose DMEM supplemented with 10\% fetal bovine serum (FBS, Biological Industries) and $1 \%$ pen-strep solution for 5 days until confluency $(>90 \%)$.

Mouse embryonic fibroblasts (MEFs) were extracted from E12.5-E14.5 Pdk total KO,

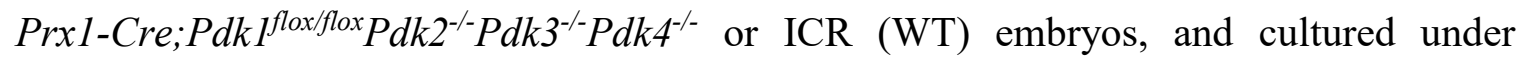
normoxic conditions in DMEM (Gibco), 20\% fetal calf serum, 1\% L-glutamine, 1\% MEMnon-essential amino acid (Biological Industries), 1\% penicillin/ streptavidin and sodium pyruvate. At passage 3 or 4, cells were harvested for either qRT-PCR, western blot analysis or viral infection. All procedures and treatments are described in Method Details.

\section{METHOD DETAILS}

\section{Viral infection}

For adeno-viral infection, MEFs were infected with 350 viral particles/cell of Ad5CMVeGFP or Ad5CMVCre-eGFP virus (Gene Transfer Vector Core, University of Iowa). MEFs were plated at a density of $500 \times 10^{3}$ cells in $6-\mathrm{cm}$ plates with the same growth medium containing the AdCMV virus for $24 \mathrm{~h}$, when medium was added. Medium was replaced after $48 \mathrm{~h}$ and cells were harvested for western blot analysis or metabolic LCMS/MS analysis 5 days post-infection. During all experiments, medium was changed daily.

\section{Histology}

For hematoxylin and eosin staining (H\&E), embryos were fixed overnight in 4\% paraformaldehyde (PFA) -phosphate-buffered saline (PBS), decalcified in a solution containing equal parts of $0.5 \mathrm{M}$ ethylenediaminetetraacetic acid (EDTA; $\mathrm{pH} 7.4$ ) and 4\% PFA in PBS overnight, dehydrated to $100 \%$ ethanol, embedded in paraffin and sectioned at a thickness of $7 \mu \mathrm{m}$. For pathological examination, sections of the entire embryo were made. H\&E staining was performed following standard protocols. 


\section{X-gal staining}

Whole-mount X-gal staining was performed as described previously (Eshkar-oren et al., 2009). In short, freshly dissected tissue was fixed in 4\% PFA/PBS, rinsed in a solution containing $5 \mathrm{mM}$ EGTA, $0.01 \%$ deoxycholate, $0.02 \% \mathrm{NP} 40$ and $2 \mathrm{mM} \mathrm{MgCl}_{2}$, and then stained in a solution containing $5 \mathrm{mM} \mathrm{K} \mathrm{K}_{3} \mathrm{Fe}(\mathrm{CN})_{6}, 5 \mathrm{mM}$ EGTA, $0.01 \%$ deoxycholate, $0.02 \% \mathrm{NP} 40,2 \mathrm{mM} \mathrm{MgCl} 2$ and $1 \mathrm{mg} / \mathrm{ml} \mathrm{X-gal}$. The tissue was cleared in $0.3 \% \mathrm{KOH}$ for better visualization.

\section{Skeletal preparation}

Cartilage and bones in whole mouse embryos or newborn pups were visualized after staining with Alcian Blue and Alizarin Red S (Sigma) and clarification of soft tissue with potassium hydroxide (Mcleod, 1980).

\section{Glucose, glutamine, glutamate and lactate measurements}

Chondrocytes were plated as described above. Upon reaching 90\% confluency, cells were washed and incubated in a glucose- and glutamine-free DMEM medium supplemented with 10\% dialyzed serum, $4 \mathrm{mM}$ L-glutamine $10 \mathrm{mM}$ glucose, for $12 \mathrm{~h}$. Subsequently, 500 $\mu \mathrm{L}$ medium from the cell culture was collected, briefly centrifuged and directly injected into the Nova chemical analyzer. Background metabolite measurements from cell-free culture were subtracted, and results were normalized to cell number.

\section{RNA isolation and quantitative real-time (qRT-) PCR}

Total RNA was purified from either MEFs or chondrocyte primary culture using the RNeasy Kit (Qiagen). Reverse transcription was performed with High Capacity Reverse Transcription Kit (Applied Biosystems) according to the manufacturer's protocol. qRTPCR was performed using Fast SYBR Green master mix (Applied Biosystems) on the StepOnePlus machine (Applied Biosystems). Values were calculated using the StepOne software version 2.2, according to the relative standard curve method. $\mathrm{Ct}$ values were 
normalized to TATA-box binding protein (Tbp) or 18S rRNA. Statistical significance was determined by Student's $t$-test as $\mathrm{P}<0.05$.

\begin{tabular}{ll}
\hline \hline Primer: & Sequence: \\
\hline \hline$P d k 1$ - forward & CGTACAGCTGGTGCAAAGTT \\
$P d k 1-$ reverse & ATTGTGCCGGTTTCTGATCC \\
$P d k 2$ - forward & GCGCTGTTGAAGAATGCGT \\
$P d k 2$ - reverse & GCCGGAGGAAAGTGAATGAC \\
$P d k 4$ - forward & CAGGGAGGTCGAGCTGTTC \\
$P d k 4$ - reverse & AGGACGTTCTTTCACAGGCA \\
$P d k 3$ - forward & TCCTGGACTTCGGAAGGGATA \\
$P d k 3$ - reverse & TCATGGTGTTAGCCAGTCGC \\
$B c k d k$-forward & AGACTTCCCTCCGATCAAGGA \\
$B c k d k$-reverse & TTTCCGGCTCTCACGAAGAC \\
$T b p-$ forward & GCAGCCTCAGTACAGCAATCAACA \\
$T b p$ - reverse & GGTGCAGTGGTCAGAGTTTGAGAA \\
$18 S$ - forward & GTAACCCGTTGAACCCCATT \\
$18 S$ - reverse & CCATCCAATCGGTAGTAGCG \\
\hline \hline
\end{tabular}

\section{Western blot analysis}

For western blotting, protein was extracted using RIPA (150mM NaCl, 1\% NP40, 0.5\% Deoxycholate, $0.1 \%$ sodium dodecyl sulfate (SDS), $50 \mathrm{mM}$ Tris $\mathrm{pH} 8$ ) supplemented with PI (1:100) and phosphatase inhibitors (1:20), homogenized using Motor Cordless (Kimble) and centrifuged at $10,000 \mathrm{rpm}, 4^{\circ} \mathrm{C}$ for $10 \mathrm{~min}$. Supernatant was collected and used for the following procedures. In the case of heart, kidney and lungs extracts were supplemented by sonication (SONICS, Vibra-Cell ${ }^{\mathrm{TM}}$ ) post-homogenization. Protein concentration was determined by Pierce protein BCA assay kit (Cyanagen). $40 \mu \mathrm{g}$ protein extracts were denatured by boiling in $\times 5$ sample buffer $(60 \mathrm{mM}$ Tris- $\mathrm{HCl}(\mathrm{pH} 6.8), 25 \%$ glycerol, $2 \%$ SDS, $14.4 \mathrm{mM} \beta$-mercaptoethanol, $0.1 \%$ bromophenol blue) for $5 \mathrm{~min}$, resolved by $10 \%$ SDS-PAGE, $100-120 \mathrm{~V}$, for $70 \mathrm{~min}$ and transferred to nitrocellulose membrane (Whatmann, 10401383) at $250 \mathrm{~mA}$ for $70 \mathrm{~min}$. Membranes were stained with Ponceau (Sigma-Aldrich, P7170) and blocked for $60 \mathrm{~min}$ at room temperature (RT) with 5\% milk$0.05 \%$ TWEEN-20. Next, membrane was incubated rocking with primary antibodies in Antibody solution ( $5 \%$ BSA fraction V, 5\% sodium azide, $0.05 \%$ PBST) overnight at $4{ }^{\circ} \mathrm{C}$. Membranes were washed $3 \times 5$ min at RT with $0.05 \%$ PBST and incubated for 60 min with horseradish peroxidase (HRP)-conjugated secondary antibodies. Membranes were washed 
$3 \times 5$ min, processed with EZ-ECL Chemiluminescence detection kit for HRP (Biological Industries, 20-500-120) and visualized by ImageQuant ${ }^{\mathrm{TM}}$ LAS 4000 (GE Healthcare Life Sciences). Densitometry values were normalized to GAPDH in the same lane.

\section{PDH activity assay}

PDH Activity Assay Kit (Sigma) was preformed according to the manufacturer's instruction. In short, protein was extracted from $\sim 10^{6} \mathrm{MEF}$ cells, homogenized using Motor Cordless (Kimble) in $100 \mu \mathrm{L}$ of ice-cold PDH Assay Buffer and centrifuged at 10,000 rpm, $4{ }^{\circ} \mathrm{C}$ for $5 \mathrm{~min}$. Supernatant was collected and used immediately ( $20 \mathrm{ul}$ per well). Colorimetric reads were done by Infinite ${ }^{\circ} 200$ PRO NanoQuant (Tecan), samples were normalized to cell count.

\section{Ketone bodies and glucose blood levels measurements}

3-beta-hydroxybutyrate (3HB), a prevalent ketone body, and glucose were measured by Freestyle Optium Neo machine (GeffenMedical) and Performa blood meter (ACCU$\mathrm{CHECK}^{\circledR}$ ), respectively, using a drop of blood from P1 Pdk total KO pups.

\section{Bioinformatic analysis of potential Pdk paralogs}

GenesLikeMe paralog analysis (https:/www.genecards.org/) for Pdkl was performed as previously described (Stelzer G, Inger A, Olender T, Iny-Stein T, Dalah I, Harel A, Safran M, 2009). Protein-protein interaction analysis was done using STRING (Szklarczyk et al., 2017).

\section{Genotype distribution analysis}

All genotype ratios were analyzed by monitoring colony progeny (4-6 months) or embryos, using tail PCR genotyping followed by Chi-square test. $\mathrm{n}$ values are given in the figure legends.

\section{Metabolite extraction}


For serum metabolite composition analysis, total blood volume (about $50 \mu 1$ ) from P1 Pdk total KO pups were collected into serum separation tubes (MiniCollect Tubes, greiner bioone) and centrifuged at $1000 \mathrm{rpm}$ for $5 \mathrm{~min}$ at RT. Serum was collected and immediate frozen in safe-lock Eppendorf tubes in liquid nitrogen. For labeled metabolites, MEF primary culture were grown and infected as described. 5 days post-infection growth medium was removed, cells were washed with pre-warmed PBS and medium was replaced with DMEM free of L-Glutamine, Glucose and Sodium Pyruvate (Biological Industries) supplemented with $2.5 \mathrm{mM}$ glucose, $2.5 \mathrm{mM}\left[3-{ }^{13} \mathrm{C}\right]$ pyruvate, $2 \mathrm{mM}$ l-glutamine and MEM-eagle non-essential amino acids $\mathrm{X} 1$, and incubated at $37^{\circ} \mathrm{C}$, for $1.5 \mathrm{~h}$. Then, medium was removed and cells were washed twice with $2 \mathrm{ml} 0.9 \%$ ice-cold saline. Cells were scraped from plates with $500 \mu \mathrm{l}$ of ice-cold methanol:DDW (1:1, v:v) containing C13 and N15 labeled amino acid mix (Sigma-Aldrich) as internal standards; a total volume of 1000 $\mu 1$ from $2 \mathrm{X}$ plates per sample (about $2 \mathrm{X} 10^{\wedge} 6$ cells) were collected into safe-lock Eppendorf tubes. Samples underwent three freeze-thaw cycles in liquid nitrogen $-37^{\circ} \mathrm{C}$ bath sonicated in ice-bath for $30 \mathrm{~min}$, and vortexed each $10 \mathrm{~min}$. Then, the samples were centrifuged for $15 \mathrm{~min}$ at maximum speed $(14000 \mathrm{rpm})$ at $4^{\circ} \mathrm{C}$. Supernatant $(800 \mu \mathrm{L})$ was transferred to another Eppendorf tube, dried for $1 \mathrm{~h}$ in speedvac and lyophilized. The dry pellet was resuspended in $100 \mu \mathrm{L}$ methanol:DDW (1:1, v:v) and centrifuged twice for $15 \mathrm{~min}$ at maximum speed $(14000 \mathrm{rpm})$ at $4{ }^{\circ} \mathrm{C}$. Then, the supernatant was transferred into vials for injection.

\section{LC-MS polar metabolites analysis}

Metabolic profiling of polar phase was done as described by Zheng et al. (2015) with minor modifications. Briefly, analysis was performed using Acquity I class UPLC System (Waters) combined with Exactive ${ }^{\mathrm{TM}}$ Plus Orbitrap Mass Spectrometer (Thermo Scientific ${ }^{\mathrm{TM}}$ ), which was operated in a negative ionization mode. The LC separation was done using the SeQuant Zic-pHilic $(150 \mathrm{~mm} \times 2.1 \mathrm{~mm})$ with the SeQuant guard column $(20 \mathrm{~mm} \times 2.1 \mathrm{~mm})($ Merck). The Mobile phase A consisted of acetonitrile and Mobile phase $\mathrm{B}$ consisted of $20 \mathrm{mM}$ ammonium carbonate plus $0.1 \%$ ammonia hydroxide in water. The flow rate was kept at $200 \mu \mathrm{min}-1$ and gradient as follow: $0-2 \mathrm{~min} 75 \%$ of B, $17 \mathrm{~min}$ $12.5 \%$ of $\mathrm{B}, 17.1 \min 25 \%$ of $\mathrm{B}, 19 \min 25 \%$ of $\mathrm{B}, 19.1 \min 75 \%$ of $\mathrm{B}, 19 \min 75 \%$ of $\mathrm{B}$. 


\section{Polar metabolites data analysis}

The data were collected using Xcalibur 4.15 and processed using Qual Browser (Thermo Scientific $\left.{ }^{\mathrm{TM}}\right)$. Compounds were identified by retention time and fragments and were verified using in-house mass spectra library. Metabolic pathway analysis was done using Metacyc database (Caspi et al., 2018).

\section{QUANTIFICATION AND STATISTICAL ANALYSIS}

Statistical analyses of qRT-PCR, chondrocyte metabolites and ketone-glucose blood levels were performed with Excel using unpaired two-tailed Student's $t$-test. PDH activity assay and metabolic profiling statistics were done using R ANOVA multiple comparison analysis. Statistical significance is denoted by asterisks $\left(\mathrm{P}<0.05[*], \mathrm{P}<0.01\left[^{* *}\right]\right.$, and $\mathrm{P}<$ $0.0001[* * *]$. The data are presented as mean $\pm \mathrm{SD}$. All statistical details, including $\mathrm{n}$ values, are given in the figures and figure legends. 


\section{REFERENCES}

1. Harris, R. A., Bowker-Kinley, M. M., Huang, B. \& Wu, P. Regulation of the activity of the pyruvate dehydrogenase complex. Adv. Enzyme Regul. 42, 249-259 (2002).

2. Tracy, B. Y., Flora, C. L. \& Reed, L. J. a-keto acid dehydrogenase complexes, x. regulation of the activity of the pyruvate dehydrogenase complex from beef kidney mitochondria by phosphorylation and dephosphorylation. 234-241 (1968).

3. O.H., W. The mammalian pyruvate dehydrogenase complex: Structure and regulation. in Reviews of Physiology, Biochemistry and Pharmacology 123-170 (Springer Berlin Heidelberg, 1983).

4. Korotchkina, L. G. \& Patel, M. S. Mutagenesis studies of the phosphorylation sites of recombinant human pyruvate dehydrogenase. Site-specific regulation. Journal of Biological Chemistry 270, 14297-14304 (1995).

5. Jeoung, N. H. Pyruvate dehydrogenase kinases: Therapeutic targets for diabetes and cancers. Diabetes Metab. J. 39, 188-197 (2015).

6. P. B. Garland, E. A. Newsholme, and P. J. R. Regulation of Glucose Uptake by Muscle. Biochem. J 93, 665-678 (1964).

7. Kolobova, E., Tuganova, a, Boulatnikov, I. \& Popov, K. M. Regulation of pyruvate dehydrogenase activity through phosphorylation at multiple sites. Biochem. J. 358, 69-77 (2001).

8. Bowker-Kinley, M. M., Davis, W. I., Wu, P., Harris, R. A. \& Popov, K. M. Evidence for existence of tissue-specific regulation of the mammalian pyruvate dehydrogenase complex. Biochem. J 329, 191-196 (1998).

9. T. E. Roche and Y. Hiromasa. Pdks regulation 2007. Cell. Mol. Life Sci 64, 830-49 (2007).

10. Sugden, M. C. \& Holness, M. J. Mechanisms underlying regulation of the expression and activities of the mammalian pyruvate dehydrogenase kinases. (2006).

doi:10.1080/13813450600935263

11. Jeong, J. Y., Jeoung, N. H., Park, K.-G. \& Lee, I.-K. Transcriptional Regulation of Pyruvate Dehydrogenase Kinase. Diabetes Metab J 36, 328-335 (2012).

12. Semenza, G. L. oxygen regulation semenza 2009. PHYSIOLOGY 24, 97-106 (2009).

13. Aragones, J., Fraisl, P., Baes, M. \& Carmeliet, P. Oxygen Sensors at the Crossroad of Metabolism. Cell Metabolism (2009). doi:10.1016/j.cmet.2008.10.001

14. Papandreou, I., Cairns, R. A., Fontana, L., Lim, A. L. \& Denko, N. C. HIF-1 mediates adaptation to hypoxia by actively downregulating mitochondrial oxygen consumption. Cell Metab. 3, 187-197 (2006).

15. Kim, J. W., Tchernyshyov, I., Semenza, G. L. \& Dang, C. V. HIF-1-mediated expression of pyruvate dehydrogenase kinase: A metabolic switch required for cellular adaptation to hypoxia. Cell Metab. (2006). doi:10.1016/j.cmet.2006.02.002

16. Kluza, J. et al. Inactivation of the HIF-1??/PDK3 signaling axis drives melanoma toward mitochondrial oxidative metabolism and potentiates the therapeutic activity of pro- 
oxidants. Cancer Res. (2012). doi:10.1158/0008-5472.CAN-12-0979

17. Lu, C. W., Lin, S. C., Chen, K. F., Lai, Y. Y. \& Tsai, S. J. Induction of pyruvate dehydrogenase kinase-3 by hypoxia-inducible factor-1 promotes metabolic switch and drug resistance. $J$. Biol. Chem. 283, 28106-28114 (2008).

18. Prigione, A. et al. HIF1?? modulates cell fate reprogramming through early glycolytic shift and upregulation of PDK1-3 and PKM2. Stem Cells (2014). doi:10.1002/stem.1552

19. Stacpoole, P. W. Therapeutic Targeting of the Pyruvate Dehydrogenase Complex / Pyruvate Dehydrogenase Kinase ( PDC / PDK ) Axis in Cancer. 109, 1-14 (2017).

20. Tennessen, J. M. et al. Coordinated Metabolic Transitions During Drosophila Embryogenesis and the Onset of Aerobic Glycolysis. doi:10.1534/g3.114.010652

21. Saunier, E., Benelli, C. \& Bortoli, S. The pyruvate dehydrogenase complex in cancer: An old metabolic gatekeeper regulated by new pathways and pharmacological agents. International Journal of Cancer (2016). doi:10.1002/ijc.29564

22. Simon, M. C. \& Keith, B. The role of oxygen availability in embryonic development and stem cell function. Nat Rev Mol Cell Biol 9, 285-296 (2008).

23. Koh, M. Y. \& Powis, G. Passing the baton: The HIF switch. Trends Biochem. Sci. 37, 364372 (2012).

24. Dunwoodie, S. L. The Role of Hypoxia in Development of the Mammalian Embryo. Developmental Cell (2009). doi:10.1016/j.devcel.2009.11.008

25. Amarilio, R. et al. HIF1alpha regulation of Sox9 is necessary to maintain differentiation of hypoxic prechondrogenic cells during early skeletogenesis. Development 134, 3917-28 (2007).

26. Provot, S. et al. Hif-1?? regulates differentiation of limb bud mesenchyme and joint development. J. Cell Biol. (2007). doi:10.1083/jcb.200612023

27. Schipani, E. et al. Hypoxia in cartilage: HIF-1?? is essential for chondrocyte growth arrest and survival. Genes Dev. 15, 2865-2876 (2001).

28. Hallmann R, Feinberg RN, Latker $\mathrm{CH}$, Sasse J, R. W. Regression of blood vessels precedes cartilage differentiation during chick limb development. Differentiation 34, 98-105 (1987).

29. Maes, C. et al. VEGF-independent cell-autonomous functions of HIF-1?? regulating oxygen consumption in fetal cartilage are critical for chondrocyte survival. J. Bone Miner. Res. 27, 596-609 (2012).

30. Bentovim, L., Amarilio, R. \& Zelzer, E. HIF1 is a central regulator of collagen hydroxylation and secretion under hypoxia during bone development. Development 139, 4473-4483 (2012).

31. Vandenboom, R. et al. compensation PDH kinase 2 knockout mice: effect of PDH kinase 1 $\mathrm{PDH}$ activation during in vitro muscle contractions in $\mathrm{PDH}$ activation during in vitro muscle contractions in PDH kinase 2 knockout mice: effect of PDH kinase 1 compensation. Am J Physiol Regul Integr Comp Physiol Am. J. Physiol. -Regulatory, Integr. Comp. Physiol. Weizmann Inst Sci (2011). doi:10.1152/ajpregu.00498.2010 
32. Ho Jeoung, N. et al. Role of pyruvate dehydrogenase kinase isoenzyme 4 (PDHK4) in glucose homoeostasis during starvation. Biochem. J 397, 417-425 (2006).

33. Nam Ho Jeoung, Yasmeen Rahim,Pengfei Wu,W. N. Paul Lee, and R. A. \& Harris. Fasting induces ketoacidosis and hypothermia in PDHK2/ PDHK4-double-knockout mice. 443, 829-839 (2012).

34. Semba, H. et al. HIF-1 $\alpha$-PDK1 axis-induced active glycolysis plays an essential role in macrophage migratory capacity. Nat. Commun. 7, 1-10 (2016).

35. Prigione, A. et al. HIF1?? modulates cell fate reprogramming through early glycolytic shift and upregulation of PDK1-3 and PKM2. Stem Cells 32, 364-376 (2014).

36. Provot, S. \& Schipani, E. Molecular mechanisms of endochondral bone development. Biochem. Biophys. Res. Commun. 328, 658-665 (2005).

37. Crabb DW, H. R. Mechanism responsible for the hypoglycemic actions of dichloroacetate and 2-chloropropionate. Arch. Biochem. Biophys. 198, 145-152 (1979).

38. Paten, B., Herrero, J., Beal, K., Fitzgerald, S. \& Birney, E. Enredo and Pecan : Genomewide mammalian consistency-based multiple alignment with paralogs. 1814-1828 (2008). doi:10.1101/gr.076554.108.

39. Stelzer, G. et al. The GeneCards Suite : From Gene Data Mining to Disease Genome Sequence Analyses. 1-33 (2016). doi:10.1002/cpbi.5

40. Broquist, H. Amino Acid Metabolism. Annu. Rev. Biochem. 35, 231-247 (1966).

41. Stelzer G, Inger A, Olender T, Iny-Stein T, Dalah I, Harel A, Safran M, L. D. GeneDecks: paralog hunting and gene-set distillation with GeneCards annotation. Omi. A J. Integr. Biol. 13, (2009).

42. Szklarczyk, D. et al. The STRING database in 2017: Quality-controlled protein-protein association networks, made broadly accessible. Nucleic Acids Res. 45, D362-D368 (2017).

43. Joshi MA, Jeoung NH, Obayashi M, Hattab EM, Brocken EG, Liechty EA, Kubek MJ, Vattem KM, Wek RC, H. R. Impaired growth and neurological abnormalities in branched-chain $\alpha$ keto acid dehydrogenase kinase-deficient mice. Biochem. J 162, 153-162 (2006).

44. Logan, M. et al. Expression of Cre Recombinase in the developing mouse limb bud driven by a Prxl enhancer. Genesis (2002). doi:10.1002/gene.10092

45. Stacpoole, P. W. The Pharmacology of Dichloroacetate. Metabolism 38, 1124-1144 (1989).

46. Zhang, S., Zeng, X., Ren, M., Mao, X. \& Qiao, S. Novel metabolic and physiological functions of branched chain amino acids: A review. J. Anim. Sci. Biotechnol. 8, 4-15 (2017).

47. Zhou, M., Lu, G., Gao, C., Wang, Y. \& Sun, H. Tissue-specific and Nutrient Regulation of the Branched-chain $\sqcup$-Keto Acid Dehydrogenase Phosphatase, Protein Phosphatase 2Cm ( PP2Cm ) * ․ 287, 23397-23406 (2012).

48. Nishitani, S. et al. Branched-chain amino acids improve glucose metabolism in rats with liver cirrhosis. 1292-1300 (2019). doi:10.1152/ajpgi.00510.2003. 
49. Shao, D. et al. Glucose promotes cell growth by suppressing branched-chain amino acid degradation. Nat. Commun. (2018). doi:10.1038/s41467-018-05362-7

50. Oppenheim, R. D. et al. BCKDH: The Missing Link in Apicomplexan Mitochondrial Metabolism Is Required for Full Virulence of Toxoplasma gondii and Plasmodium berghei. PLoS Pathog. 10, (2014).

51. Wynn, R. M., Chuang, J. L., Cote, C. D. \& Chuang, D. T. Tetrameric Assembly and Conservation in the ATP-binding Domain of Rat Branched-chain $\backsim$-Ketoacid Dehydrogenase Kinase *. 275, 30512-30519 (2000).

52. Gudi, R. et al. Diversity of the Pyruvate Dehydrogenase Kinase Gene Family in Humans. Cell Biol. Metab. 270, 28989-28994 (1995).

53. K M Popov, N Y Kedishvili, Y Zhao, Y Shimomura, D. W. C. and R. A. H. Primary Structure of Pyruvate Dehydrogenase Kinase Establishes a New Family of Eukaryotic Protein Kinases *. J. Biol. Chem. 268, 26602-26606 (1993).

54. Ferriero, R. et al. Phenylbutyrate Therapy for Pyruvate Dehydrogenase Complex Deficiency Phenylbutyrate Therapy for Pyruvate Dehydrogenase Complex Deficiency and Lactic Acidosis. 175, (2013).

55. Huang, B. et al. Isoenzymes of Pyruvate Dehydrogenase Phosphatase. J. Biol. Chem. 273, 17680-17688 (1998).

56. Semenza, G. L. Hypoxia-inducible factor 1 : oxygen homeostasis and disease pathophysiology. TRENDS Mol. Med. 7, 345-350 (2001).

57. Kim, J. W., Tchernyshyov, I., Semenza, G. L. \& Dang, C. V. HIF-1-mediated expression of pyruvate dehydrogenase kinase: A metabolic switch required for cellular adaptation to hypoxia. Cell Metab. 3, 177-185 (2006).

58. Weissgerber, T. L. \& Wolfe, L. A. Physiological adaptation in early human pregnancy : adaptation to balance maternal - fetal demands. 11, 1-11 (2006). 


\section{BCKDK regulates the TCA cycle through PDC to ensure embryonic development in the absence of PDK family}

Lia Heinemann-Yerushalmi, ${ }^{* 1}$ Lital Bentovim, ${ }^{* 1}$ Neta Felsenthal, ${ }^{1}$ Ron Carmel Vinestock, ${ }^{1}$ Nofar Michaeli, ${ }^{1}$ Sharon Krief, ${ }^{1}$ Alon Silberman, ${ }^{5}$ Marina Cohen, ${ }^{2}$ Shifra Ben-Dor, ${ }^{3}$ Ori Brenner, ${ }^{2}$ Rebecca Haffner-Krausz, ${ }^{2}$ Maxim Itkin, ${ }^{4}$ Sergey Malitsky, ${ }^{4}$ Ayelet Erez ${ }^{5}$ and Elazar Zelzer ${ }^{1}$

${ }^{1}$ Department of Molecular Genetics, Weizmann Institute of Science, 234 Herzl St, Rehovot 7610001, Israel

${ }^{2}$ Department of Veterinary Resources, Weizmann Institute of Science, 234 Herzl St, Rehovot 7610001, Israel

${ }^{3}$ Bioinformatics and Biological Computing Unit, Biological Services, Weizmann Institute of Science, Rehovot, 76100, Israel

${ }^{4}$ Department of Life Sciences Core Facilities, Weizmann Institute of Science, Rehovot, Israel

${ }^{5}$ Department of Biological regulation, Weizmann Institute of Science, Rehovot, Israel *These authors equally contributed to this paper.

\section{Correspondence:}

Elazar Zelzer eli.zelzer@weizmann.ac.i1 

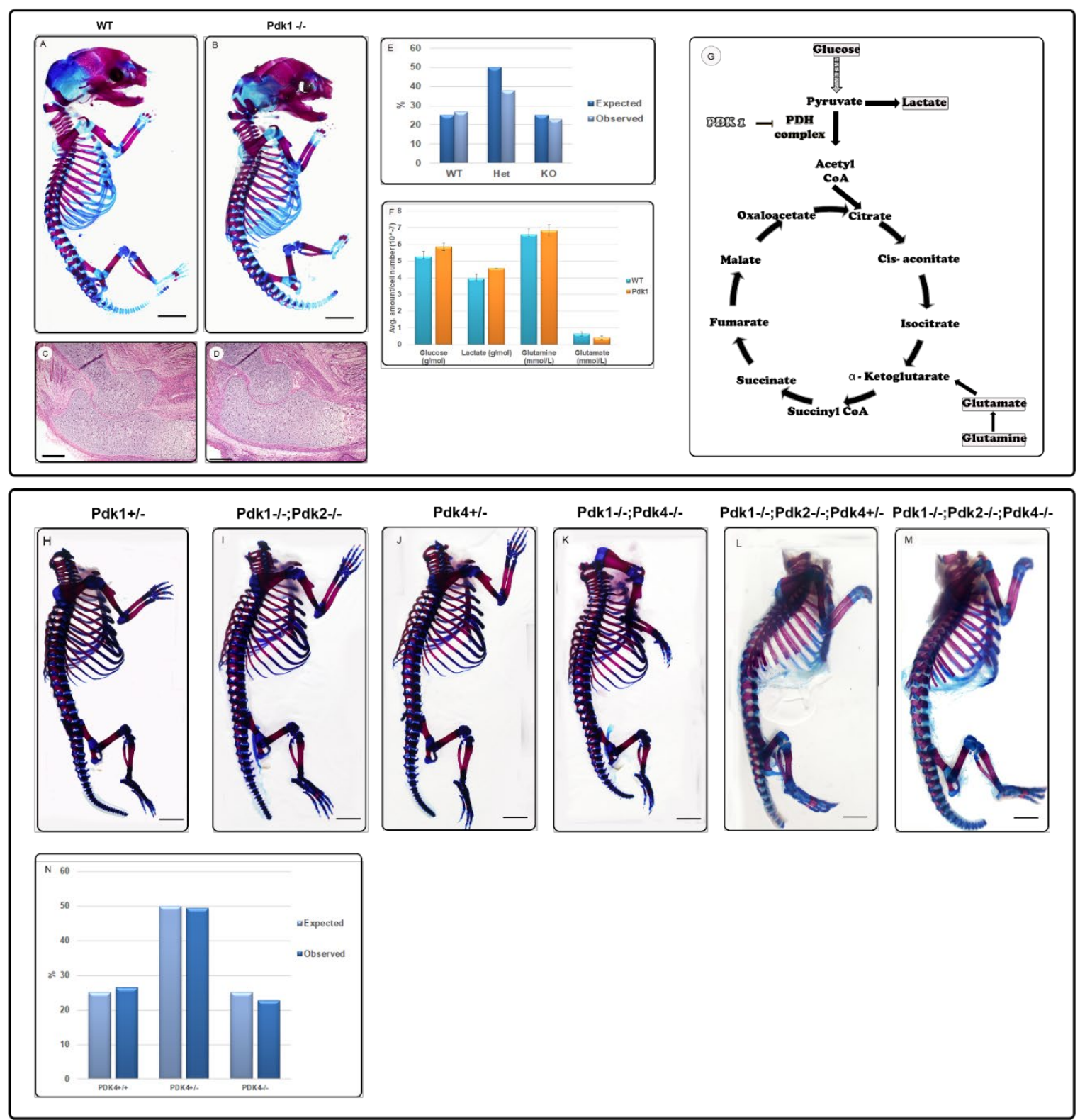

Figure 1. Deletion of single, double or triple Pdk genes in vivo does not affect embryonic development. (A,B) Skeletal preparations of control (A) and Pdk1 KO (B) E17.5 embryos showing no skeletal phenotype (scale, $50 \mu \mathrm{m})$. (C,D) H\&E staining of control and Pdk1 KO E15.5 embryonic sections of olecranon and distal humerus growth plates (scale, $50 \mu \mathrm{m}$ ). (E) Graph showing expected Mendelian ratios of Pdk1 KO progeny. (F) Graph showing similar absolute levels of glycolysis-TCA pathway-related metabolites in chondrocytes from E17.5 Pdk1 KO and WT embryos (Nova analyzer, n=3). (G) Schematic illustration of the TCA cycle highlighting the relevant metabolites entry point to the cycle . (H-M) Skeletal preparations of control (Pdk1+/-, H), Pdk1-Pdk2 dKO (I), control (Pdk4+/-, J) and Pdk1-Pdk4 Dko (K), control (Pdk1-/- 
bioRxiv preprint doi: https://doi.org/10.1101/2020.03.22.002360; this version posted December 17, 2020. The copyright holder for this preprint (which was not certified by peer review) is the author/funder. All rights reserved. No reuse allowed without permission.

Pdk2-/- Pdk4+/-,l) and Pdk1-Pdk2-Pdk4 tKO (M) newborn pups. (H and I, J and K, and L and M are littermates; scale, $50 \mu \mathrm{m})$. (N) Graph showing expected Mendelian ratios in colonies of Pdk tKO mice./ 


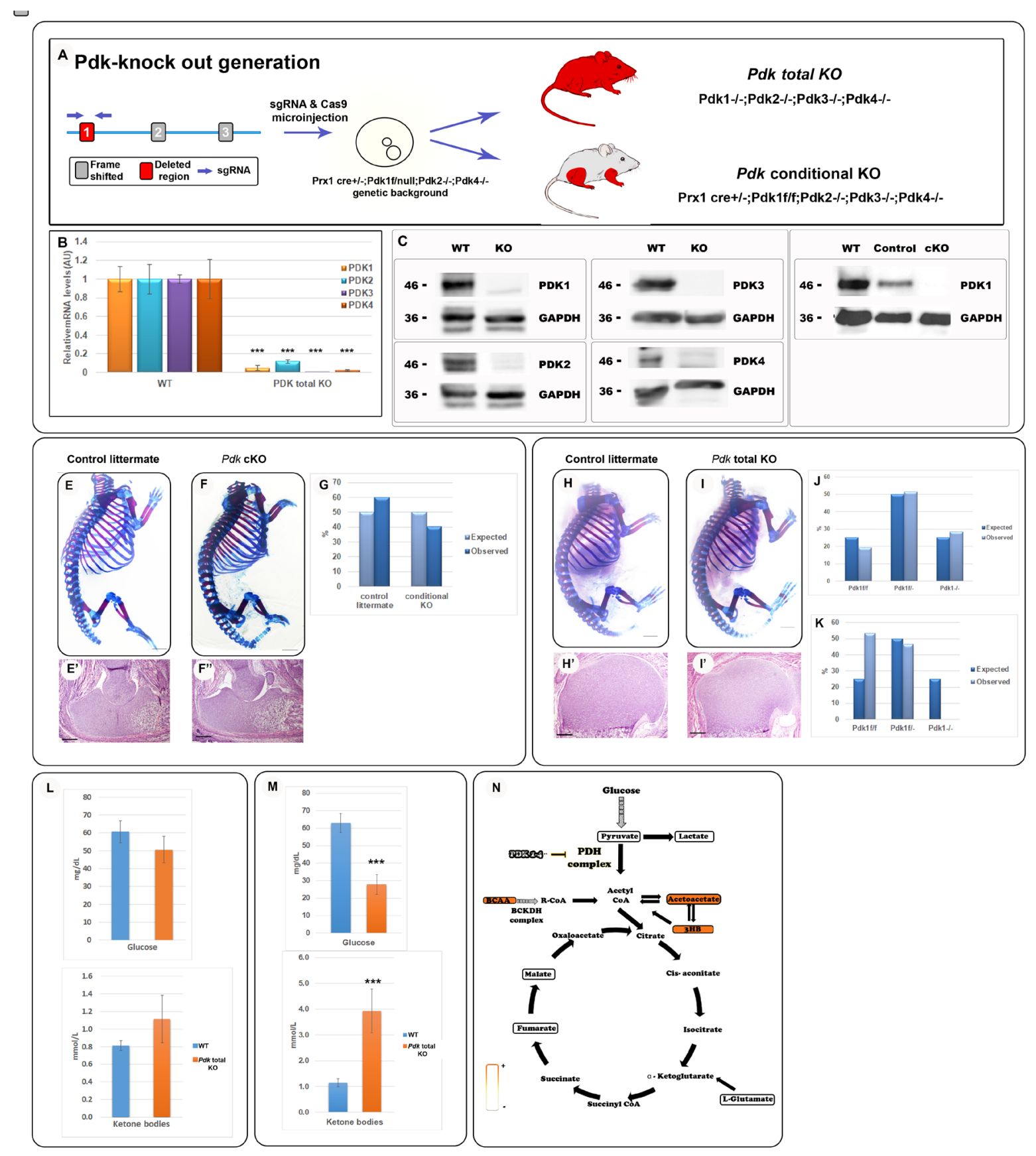

Figure 2. Deletion of the entire $\boldsymbol{P d} \boldsymbol{k}$ family results in postnatal lethality. (A) Schematic representation of $P d k 3$ gene and the targeted site of CRISPR/Cas9-mediated deletion. This was followed by zygote injection to generate $P d k$ total KO mice or $P d k$ conditional KO in limb mesenchyme only, depending on the genetic background. (B) qRT-PCR of Pdk1-4 mRNA confirms deletion of all four genes, as compare to WT (***, $\mathrm{P}<0.0001 ; \mathrm{n}=4$ for each genotype; data are normalized to Tbp and presented as mean $\pm \mathrm{SD}$ ). (C) Protein expression analysis (western blot) of four PDK isoenzymes in heart samples derived from $P d k$ total KO or WT P0 pups shows complete deletion of the entire PDK family (representative images, n=3). (D) Western blotting of chondrocytes from Pdk cKO, control littermate and WT embryos shows deletion of PDK1 in the mutant (representative images, 3 biological repeats, $n=5$ for each sample). (E-F') Skeletal preparations (E,F) 
and H\&E-stained olecranon and distal humerus sections (E',F') from E18.5 Prxl-Cre;Pdk ${ }^{f f f} ; P d k 2^{--} ; P^{\prime} d k^{-/-}$ ;Pdk4 $4^{-\kappa}$ embryos and control littermates (without Cre) show that the $P d k$ cKO embryos develop normally (scale, $100 \mu \mathrm{m}$ ). (G) Graph showing Mendelian ratios of genotypes in Pdk cKO progeny. (H-I') Skeletal preparations at E18.5 (H-I) and H\&E-stained sections (H',I') from P0 Prxl-Cre;Pdk $1^{1 /-} ; P d k 2^{-/} ; P d k 3^{-/} ; P d k 4^{-}$ ^ mice and control littermates (scale, $100 \mu \mathrm{m}$ ). (J) Graph showing Mendelian ratios of genotypes in E18.5 $P d k$ total KO embryos. (K) Graph showing non-Mendelian ratios of genotypes in Pdk total KO mature mice, as a result of loss of the total $\mathrm{KO}$ genotype. (L) Graphs showing in utero blood levels of glucose and $3 \mathrm{HB}$, a ketone body, in $P d k$ total KO E18.5 embryos and P1 pups (M), compared to WT. Data are presented as mean \pm SE (embryos: $\mathrm{n}_{\mathrm{WT}}=9, \mathrm{n}_{\mathrm{KO}}=6 ; \mathrm{P} 1: \mathrm{n}_{\mathrm{WT}}=9 ; \mathrm{n}_{\mathrm{KO}}=4$, from 3 independent litters; $\mathrm{P}<0.05$, Student's $t$-test). (N) Scheme of serum metabolic profiles of P1 Pdk total KO pups, as determined by LC-MS polar metabolite analysis. Higher levels are shown in orange and lower levels in yellow, as compared to WT levels $(\mathrm{P}<0.05$; fold change $>2, \mathrm{n}_{\mathrm{WT}}=4 ; \mathrm{n}_{\mathrm{KO}}=3$ ). 

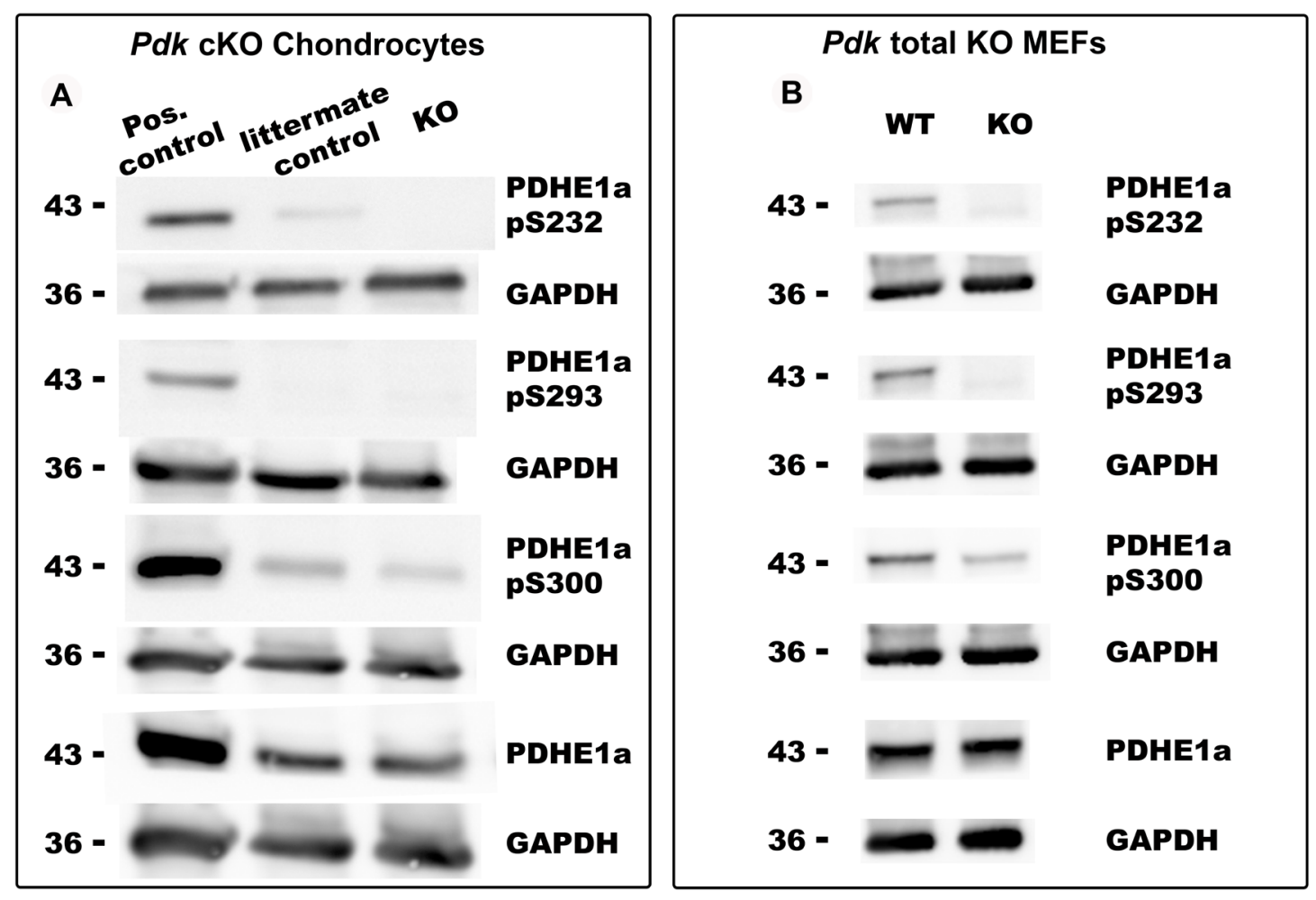

Figure 3. PDC phosphorylation is PDK-independent. Western blot analysis of the three PDC phosphorylation sites in chondrocytes extracted from growth plates of E17.5 Pdk cKO embryos (A; Prx1CrePdk $1^{\text {floxflox }} P d k 2^{-/-} P d k 3^{-/-} P d k 4^{-/-}$) and MEFs derived from $P d k$ total $\mathrm{KO}$ embryos (B), compared to WT. As controls, PDH1a and endogenous GAPDH total protein levels were measured. Each chondrocyte sample is a pool of 5 biological repeats; $n=3$ for all groups. Hearts of E17.5 WT embryos are shown as a positive control, littermate control were Prx1-Cre-negative. MEF samples, $\mathrm{n}=7$. 

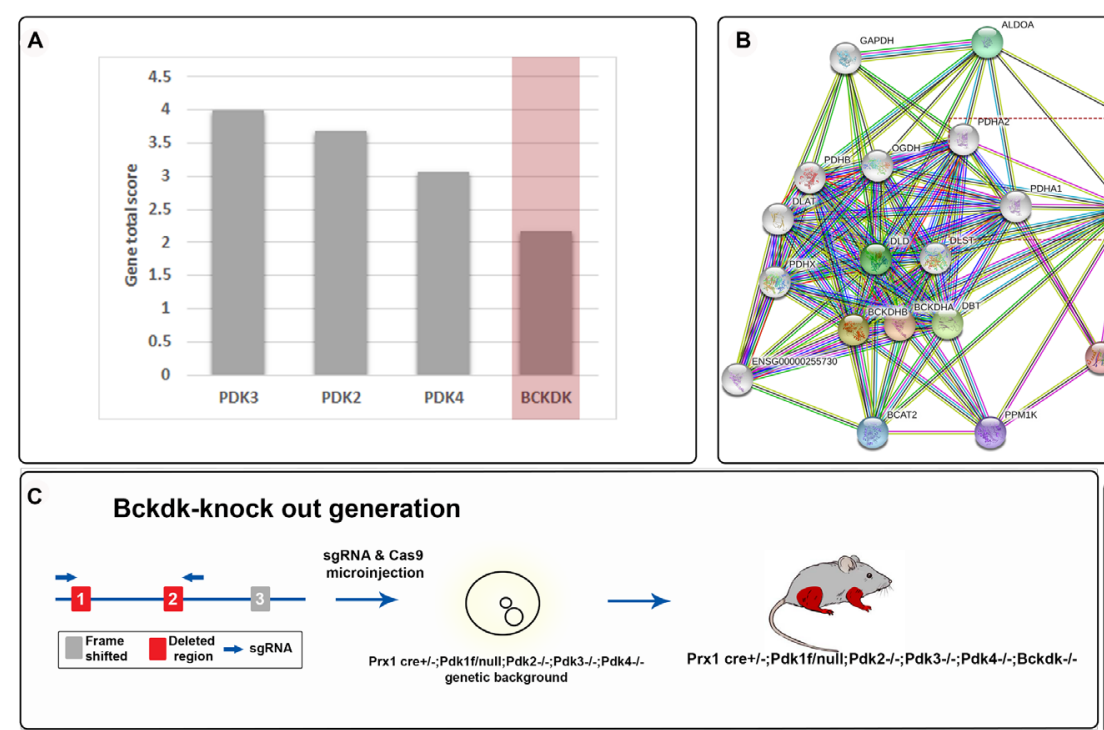

\begin{tabular}{|c|c|c|c|c|}
\hline \multirow{2}{*}{\multicolumn{5}{|c|}{ F Prx1 cre+:Pdk1f/f;2;3;4KO;Bck+/- x Pdk1f/f;2;3;4KO;Bck+/- }} \\
\hline & & & & \\
\hline Genotype & Expected (\%) & \multicolumn{2}{|c|}{ Observed (\%) } & Expected \\
\hline Pdk1f/f;2;3;4KO;Bck WT & $5.3(12.5 \%)$ & \multicolumn{2}{|c|}{$6(14.6 \%)$} & $3.9(12.5$ \\
\hline Pdk1f/f;2;3;4KO;Bck het & $10.5(25 \%)$ & \multicolumn{2}{|c|}{$12(29.3 \%)$} & $7.8(25 \%$ \\
\hline Pdk1f/f;2;3;4KO;Bck KO & $5.3(12.5 \%)$ & \multicolumn{2}{|c|}{$3(7.3 \%)$} & 3.9 (12.5 \\
\hline Prx1 cre:Pdk1f/f;2;3;4KO;Bck WT & $5.3(12.5 \%)$ & \multicolumn{2}{|c|}{$5(12.2 \%)$} & $3.9(12.5$ \\
\hline Prx1 cre:Pdk1f/f;2;3;4KO;Bck het & $10.5(25 \%)$ & \multicolumn{2}{|c|}{$11(26.8 \%)$} & $7.8(25 \%$ \\
\hline Pr×1 cre:Pdk1f/f;;;3;4KO;Bck KO & $5.2(12.5 \%)$ & \multicolumn{2}{|c|}{$4(9.8 \%)$} & 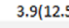 \\
\hline Total & \multirow{2}{*}{\multicolumn{3}{|c|}{$\begin{array}{r}n=41 \\
0.890 \\
\end{array}$}} & \\
\hline chi-Squaretest & & & & \\
\hline \multicolumn{5}{|c|}{ H Prx1 cre+:Pdk1fff;2;3;4KO;Bck+/- x Pdk1f/f;2;3;4KO;Bck -/- } \\
\hline Genotype & \multicolumn{4}{|c|}{ Expected (\%) Observed (\%) } \\
\hline Pdk1f/f;2;3;4;Bck het & \multicolumn{2}{|c|}{$4.8(25 \%)$} & \multicolumn{2}{|c|}{$12(46 \%)$} \\
\hline Pdk1f/f;2;3;4;Bck KO & \multicolumn{2}{|c|}{$4.8(25 \%)$} & \multicolumn{2}{|c|}{$9(35 \%)$} \\
\hline Prx1 cre;Pdk1f/f;2;3;4;Bck het & \multicolumn{2}{|c|}{$4.8(25 \%)$} & \multicolumn{2}{|c|}{$5(19 \%)$} \\
\hline Prх1 cre;Pdk1f/f;2;3;4;Bck КО & & 25\%) & & $\%)$ \\
\hline Total & \multicolumn{4}{|c|}{$\mathrm{N}=26$} \\
\hline Chi-Square test & \multicolumn{4}{|c|}{$2.02 \mathrm{E}-04$} \\
\hline
\end{tabular}
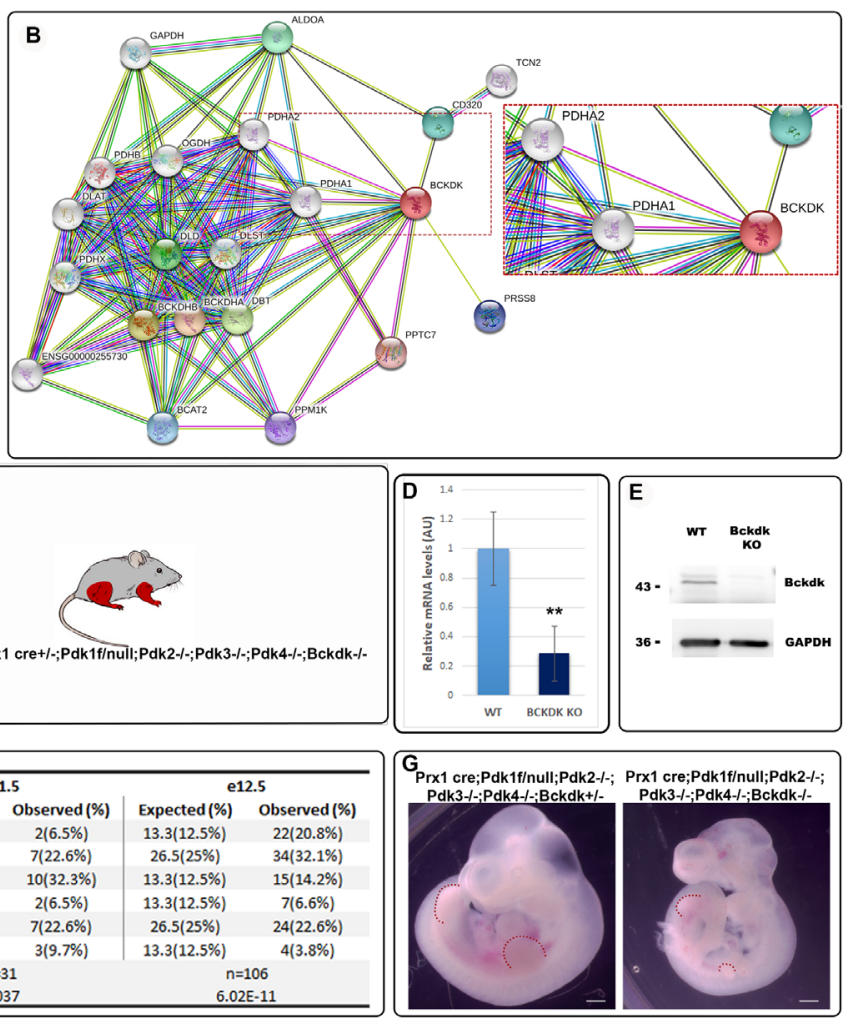

$n=31$
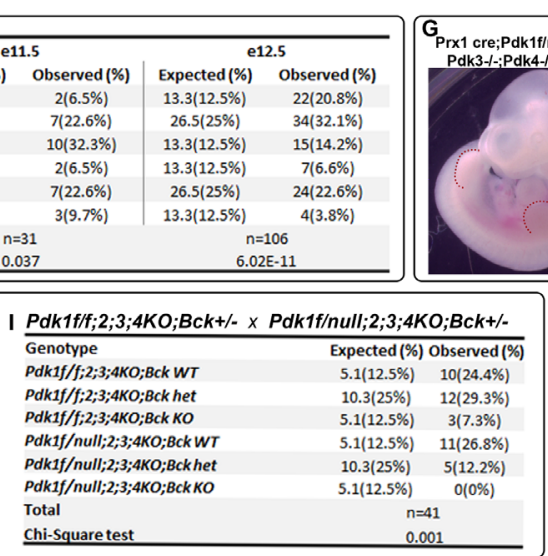

Figure 4. Prx1-CrePdk cKO-Bckdk KO mice display embryonic lethality. (A) GenesLikeMe analysis identifies $B c k d k$ as a $P d k 1$ paralog, along with the other $P d k$ family members (total score combines sequences, domains, super pathways, expression, compounds and gene ontology similarities). (B) STRING analysis predicts protein-protein interaction between BCKDK and PDH complex subunit PDH1a (string legend: yellow, textmining; black, co-expression; pink, experimentally determined). (C) Schematic representation of $B c k d k$ gene and the targeted sites of CRISPR/Cas9-mediated deletion. This was followed by zygote injection to generate Prx 1-Cre-Pdkf flox/null $P d k 2^{-/} P d k 3^{-/-} 4^{-/-} B c k d k^{-/}$mouse, lacking $P d k 1$ in limb mesenchyme lineages. (D) qRT-PCR of Bckdk mRNA in Prxl-Cre-Pdkl flox/null $P d k 2^{-/} P d k 3^{-/-} 4^{-/}$ $B c k d k^{-/}$mice, as compare to WT (**, $\mathrm{P}<0.001 ; \mathrm{n}=3$ for each genotype; data normalized to $T b p$ and presented as mean \pm SD). (E) Protein expression analysis by western blotting shows complete deletion of BCKDK in $P d k 1^{\text {floxflox }} P d k 2^{-/} P d k 3^{-/} 4^{-/} B c k d k^{-/}$MEF cells (n=3). (F) Expected Mendelian ratios and observed genotype distribution of Prxl-Cre-Pdk $1^{\text {floxflox }} P d k 2^{-/} P d k 3^{-/-} 4^{-/} B c k d k^{+-}$crossed with $P d k 1^{\text {floxflox }} P d k 2^{-/-}$ $P d k 3^{-/-} 4^{-/} B c k d k^{+/}$embryos at E10.5 (n=41), E11.5 (n=31) and E12.5 (n=106). (G) Representative E10.5 Prx 1-Cre-Pdk1 floxflox $P d k 2^{-/-} P d k 3^{-/} 4^{-/} B c k d k^{--}$embryo displays delayed development as compared to 
control $B c k d k$ heterozygous littermate (dashed red lines indicate the limbs; scale, $50 \mu \mathrm{m}$ ). (H) Mendelian

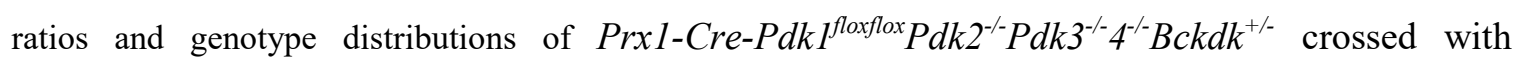
$P d k f^{f l o x f l o x} P d k 2^{-/} P d k 3^{-/-} 4^{-/} B c k d k^{-/}$mice and (I) $P d k 1^{f l o x / n u l l} P d k 2^{-/} P d k 3^{-/} 4^{-/} B c k d k^{+/-}$crossed with $P d k f^{f l o x / m u l l} P d k 2^{-/} P d k 3^{-/-} 4^{-/-} B c k d k^{+/}$mice (postnatal stage, after weaning). Data are presented as numbers and percentage of the total progeny, statistical significance was determined by Chi-square test. 

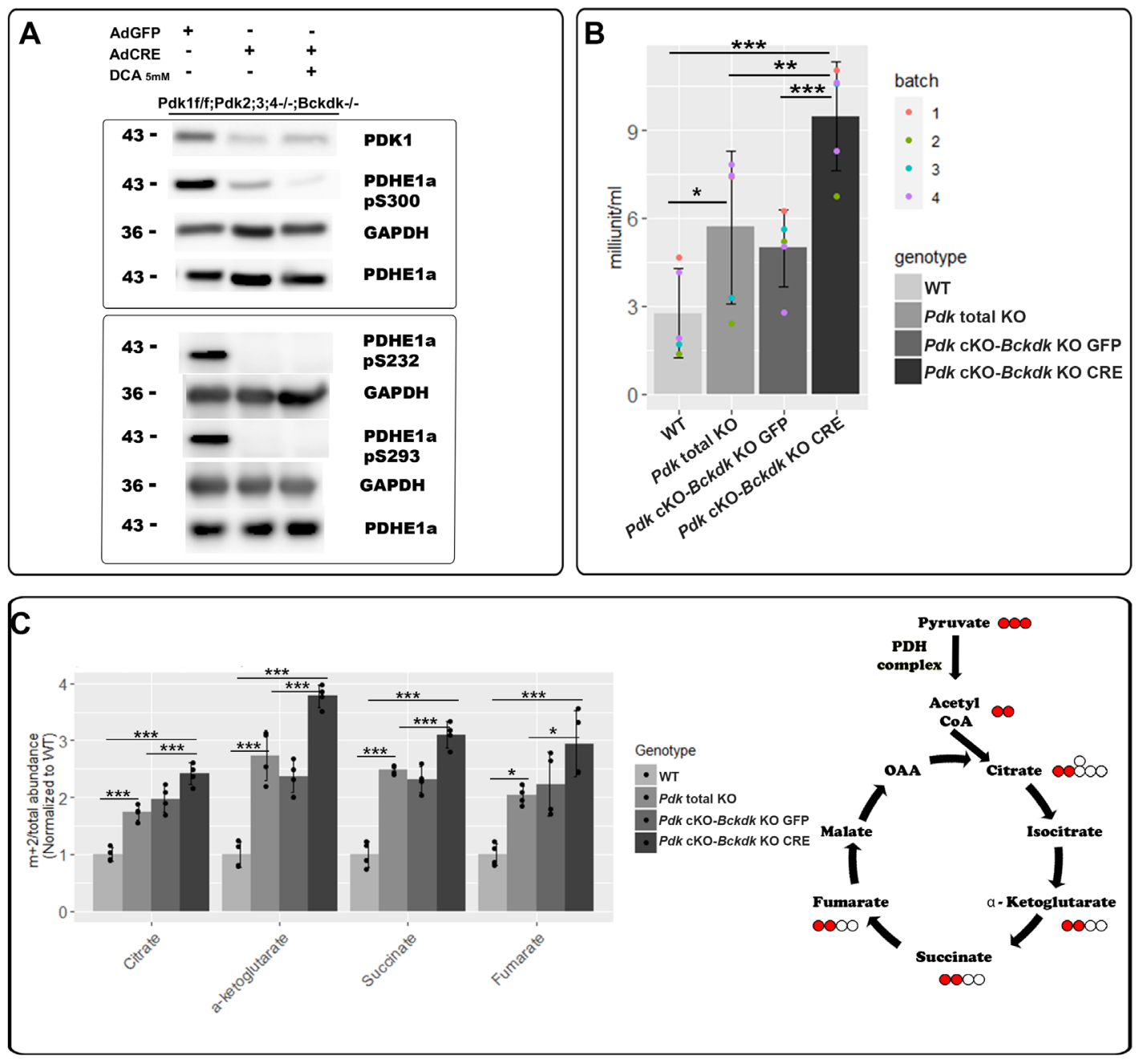

Figure 5. PDC phosphorylation and activity is BCKDK-dependent. (A) Western blot of PDK1 and of PDH1a phosphorylated sites pS300, the main active PDC site in MEFs, pS232 and pS293

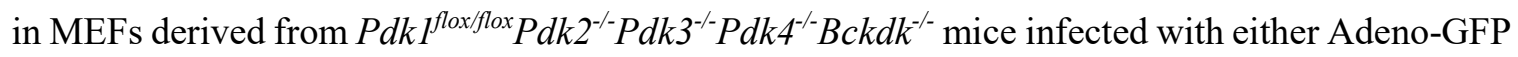
as a control, or Adeno-CRE with or without addition of $5 \mathrm{mM}$ DCA, to ablate PDK1 (representative images, $\mathrm{n}=6$ from two different sets of experiments). (B) PDC activity assay in MEFs from $P d k$

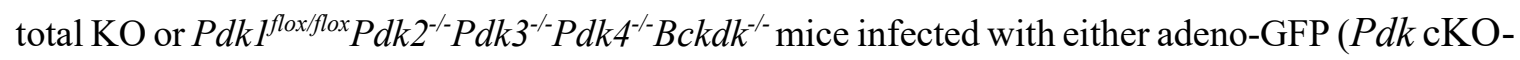
$B c k d k \mathrm{KO}$ GFP) or adeno-Cre ( $P d k \mathrm{cKO}-B c k d k \mathrm{KO} \mathrm{CRE})$, and WT as a control (mean $\pm \mathrm{SD}$, *** $\mathrm{P}<0.001, * * \mathrm{P}<0.01, * \mathrm{P}<0.05, \mathrm{n}=5$ for each genotype from 4 different experiments, ANOVA multiple comparison test). (C) Analysis of $\left[3-{ }^{13} \mathrm{C}\right]$ pyruvate flux into the TCA cycle using

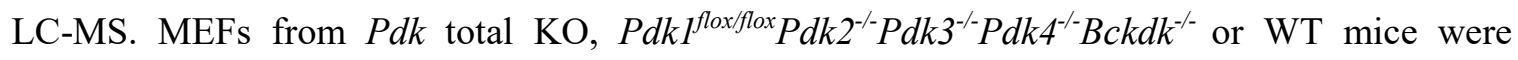
infected with either adeno-GFP ( $P d k \mathrm{cKO}-B c k d k \mathrm{KO}$ GFP) or adeno-Cre ( $P d k \mathrm{cKO}-B c k d k \mathrm{KO}$ CRE). Graph shows the fraction of $m+2$ mass isotopomers of TCA cycle intermediates from the total abundance of these metabolites, normalized to WT levels. On the right is a schematics of the 
bioRxiv preprint doi: https://doi.org/10.1101/2020.03.22.002360; this version posted December 17, 2020. The copyright holder for this preprint (which was not certified by peer review) is the author/funder. All rights reserved. No reuse allowed without permission.

TCA cycle showing the labeled metabolites (mean $\pm \mathrm{SD}, * * * \mathrm{P}<0.001, * * \mathrm{P}<0.01, * \mathrm{P}<0.05$, $\mathrm{n}=4$ for each genotype, ANOVA multiple comparison test). 